Research Article

\title{
Antidisturbance Control for Helicopter Stochastic Systems
}

\author{
Dongping $\mathrm{Li}^{1}$ and Yankai $\mathrm{Li} \mathbb{D}^{2}$ \\ ${ }^{1}$ School of Sciences, Xi'an Technological University, Xi'an 710021, China \\ ${ }^{2}$ Institute of Automation and Information Engineering, Xi'an University of Technology, Xi'an 710048, China
}

Correspondence should be addressed to Yankai Li; liyankai@xaut.edu.cn

Received 25 August 2021; Revised 6 October 2021; Accepted 15 October 2021; Published 16 November 2021

Academic Editor: Akif Akgul

Copyright $\odot 2021$ Dongping Li and Yankai Li. This is an open access article distributed under the Creative Commons Attribution License, which permits unrestricted use, distribution, and reproduction in any medium, provided the original work is properly cited.

\begin{abstract}
In this paper, an antidisturbance controller is presented for helicopter stochastic systems under disturbances. To enhance the antidisturbance abilities, the nonlinear disturbance observer method is applied to reject the time-varying disturbances. Then, the antidisturbance nonlinear controller is designed by combining the backstepping control scheme. And the stochastic theory is used to guarantee that the closed-loop system is asymptotically bounded in mean square while the proposed control method is shown via some traditional nonlinear control techniques, which still show some common issues such as "dimension explosion" or others. The result of this paper can be regarded as a typical case of the nonlinear control method to help and promote the generation of advanced methods.
\end{abstract}

\section{Introduction}

For actual systems, the nonlinearities of the model are probably one of the most noticeable characteristics, which are issued from the physical laws, material science, mathematical derivation, and so on. Hence, nonlinear systems are typical research models in various control fields and can be used to describe the dynamics of the system states. In order to handle nonlinear control problems, many classical control methods were proposed based on the nonlinear control theory [1-3], such as the baskstepping control method, feedback linearization technique, and nonlinear disturbance observer-based control scheme. Based on these nonlinear control theories, a number of advanced control techniques were proposed for various control systems. In [4], a class of nonlinear systems was studied via the event-triggered robust adaptive fuzzy control method. In [5], the adaptive neural control scheme was adopted for the nonlinear multiple output systems under the time-varying output constraints. Combining the nonlinear disturbance observer control with sliding-mode fuzzy neural network methods, a new control scheme was presented to deal with the nonlinear systems with disturbances in [6]. In $[7,8]$, the issue of adaptive fuzzy tracking control was discussed for a class of strict-feedback nonlinear systems. In [9], the periodic event-triggered control method was used to design the controller for nonlinear networked control systems. In [10], the robust adaptive control problem was investigated for state-constrained nonlinear systems under the input saturation and unknown control direction. The observer-based $H_{\infty}$ control was used for discrete-time T-S fuzzy systems in [11]. In [12], the switched-observer-based adaptive output-feedback control scheme was designed for pure-feedback switched nonlinear systems with unknown gains. The model-based adaptive event-triggered control method was discussed for nonlinear continuous-time systems in [13]. From the above discussion, the nonlinear control methods and theories are effective for disposing many troublesome nonlinear control problems. Therefore, the nonlinear control system theories are useful approaches to solve the issues of flight control for helicopters.

The flight control is a crucial issue of helicopters. An excellent flight controller guarantees the well flight performances of flying helicopters and prevents the crash, breakdown, and so on. In many books involving helicopters $[14,15]$, linear control methods were used for designing the helicopter flight controller and obtained expected control performances while, in fact, helicopter models are 
complicated nonlinear systems, which are constructed according to the relationships of the components of helicopters. In some outstanding results, the helicopter flight controllers are designed by using the linear models, which are evolved from the nonlinear helicopter models under the linearization techniques. In [16], the tracking control issue was discussed for small-scale unmanned helicopter linear systems. In [17], the flight controller was designed for helicopters via the probabilistic robust linear parametervarying control method using the iterative scenario approach. In [18], the model reference resilient control methods were designed for linear helicopter systems with time-varying disturbances. In [19], tracking control issues were discussed for the linear helicopter systems under timevarying disturbances and input stochastic perturbation. However, the dynamics of the helicopter states are complicated with many nonlinear characteristics. With development of the helicopter flight control techniques, the nonlinear flight controllers are designed for various helicopter systems. In [20], the adaptive trajectory tracking control approach was proposed for model-scaled nonlinear helicopter systems. The trajectory tracking control problems were discussed for small-scale nonlinear unmanned helicopters under model uncertainties in [21]. In [22], the composite block backstepping trajectory tracking control scheme was presented for disturbed nonlinear unmanned helicopter systems. In [23], the fixed-time autonomous shipboard landing control issues were studied for nonlinear helicopter systems under external disturbances. The finitetime control issue was discussed for small-scale nonlinear unmanned helicopter systems with disturbances in [24]. In [25], the sliding-mode control and extended disturbance observer methods were used to solve the tracking control issue of unmanned nonlinear helicopter systems. The dynamic decoupling control optimization methods were adopted for small-scale nonlinear unmanned helicopter systems in [26]. While helicopters fly in full noise surroundings, various random disturbances exist in helicopter control system models. In [27], the antidisturbance control was proposed for the attitude and altitude nonlinear helicopter systems under random disturbances. The random disturbances are considered for flight control, which improves the control performances and precision of flying helicopters.

In this paper, in order to enhance the control precision and robustness of the helicopter models, the random disturbances are considered to construct the fight controller. The helicopter stochastic systems are better to describe the flying dynamics states of the helicopter. While with the random disturbances introducing in the models, many advanced control methods, such as dynamic surface control, fuzzy control, and other intelligent control methods, cannot be used to design the flight controller directly because many control variables will lose some good characteristics, such as the continuity and derivability, under the random disturbances. Therefore, in this manuscript, the backstepping control method and nonlinear disturbance observer control scheme are used to establish the nonlinear flight controller. Due to the characteristics of underactuation, strong nonlinearity, and high-order of helicopter stochastic system models, the phenomenon of "dimension explosion" is inevitable for nonlinear controller designed, and the controller solved steps are presented in this paper. Our results present an approach to design the strong robust flight controller.

The organization of this paper is standard. The problem statement is stated in Section 2. The position loop control issues are discussed in Section 3. The attitude loop control problems are studied in Section 4. The stability analysis of the main result is given in Section 5. The conclusions are presented in Section 6.

\section{Problem Statement}

Consider the following helicopter dynamic system:

$$
\begin{aligned}
\mathrm{d} p & =v \mathrm{~d} t, \\
\mathrm{~d} v & =\left(-g e_{3}+\frac{1}{m} R f\right) \mathrm{d} t+\delta_{1} \mathrm{~d} t+G_{1} \mathrm{~d} \beta_{1}, \\
\mathrm{~d} R & =R w^{\times} \mathrm{d} t, \\
J \mathrm{~d} w & =\left(-w^{\times} J w+\tau\right) \mathrm{d} t+\delta_{2} \mathrm{~d} t+G_{2} \mathrm{~d} \beta_{2},
\end{aligned}
$$

where $p$ and $v$ denote the position and velocity in inertial frame, respectively, $g$ is the gravitational acceleration, $e_{3}=$ $\left(\begin{array}{lll}0 & 0 & 1\end{array}\right)^{T}$ is a unit vector, and $R$ is the rotation matrix from body frame to inertial frame and defined by

$$
\begin{aligned}
R & =\left(\begin{array}{ccc}
C \theta C \psi & S \theta S \phi C \psi-C \phi S \psi & C \phi S \theta C \psi+S \phi S \psi \\
C \theta S \psi & S \phi S \theta S \psi+C \phi C \psi & C \phi S \theta S \psi-S \phi C \psi \\
-S \theta & S \phi C \theta & C \phi C \theta
\end{array}\right) \\
& =\left(\begin{array}{lll}
R_{11} & R_{12} & R_{13} \\
R_{21} & R_{22} & R_{23} \\
R_{31} & R_{32} & R_{33}
\end{array}\right)=\left(\begin{array}{lll}
R_{1} & R_{2} & R_{3}
\end{array}\right),
\end{aligned}
$$

where $C$. and $S \cdot$ denote $\cos (\cdot)$ and $\sin (\cdot)$ with respective variables. $\phi(t), \theta(t)$, and $\varphi(t)$ are roll, pitch, and yaw angles in body frame, respectively. $w=(p(t) q(t) r(t))$ is the angular velocity. $p, q$, and $r$ are the roll, pitch, and yaw angular rates, respectively. $J$ is the inertia matrix and denoted by $J=\operatorname{diag}\left\{\begin{array}{llll}J_{x x} & J_{y y} & J_{z z}\end{array}\right\} . f$ and $\tau$ are the sum of the external forces and moments. Consider the characteristics of these forces and moments, which are denoted as follows: 


$$
\begin{aligned}
& f=\left(\begin{array}{lll}
0 & 0 & T_{m}
\end{array}\right)^{T}, \\
& \tau_{u}=\left(\begin{array}{c}
C_{m}+T_{m} L_{z}-H_{z} \\
C_{m}+T_{m} L_{z} 00 \\
-T_{m} L_{y} T_{m} L_{x} H_{x}
\end{array}\right)\left(\begin{array}{c}
a \\
b \\
T_{t}
\end{array}\right)+\left(\begin{array}{c}
-T_{m} L_{y} \\
-T_{m} L_{x} \\
-Q_{m}
\end{array}\right)=A\left(T_{m}\right) \rho+B\left(T_{m}\right),
\end{aligned}
$$

with $Q_{m}=C_{k} T_{m}^{1.5}+D_{k} . C_{m}, L_{z}, H_{z}, L_{y}, L_{x}, H_{x}$, and $D_{k}$ are known constants. $\beta_{1}$ and $\beta_{2}$ are independent one-dimensional standard Wiener processes, $\left(\mathrm{d} \beta_{1} / \mathrm{d} t\right)=\xi_{1}$ and $\left(\mathrm{d} \beta_{2} / \mathrm{d} t\right)=\xi_{2}$, where $\xi_{1}$ and $\xi_{2}$ are independent one-dimensional stochastic noises. $G_{1} \xi_{1}$ and $G_{2} \xi_{2}$ are the stochastic accelerated velocity and stochastic angular acceleration generated by the stochastic force and stochastic moment, $G_{1}$ and $G_{2}$ are known and bounded weight parameters, and $\delta_{1}$ and $\delta_{2}$ are disturbances with bounded derivatives, which are $\left\|\dot{\delta}_{1}\right\| \leq \mu_{1}$ and $\left\|\dot{\delta}_{2}\right\| \leq \mu_{2}$.

The following definitions and lemmas are crucial important to further analyze the main results of this paper. In order to state those definitions and lemmas, consider the following system:

$$
\mathrm{d} x=f(t, x) \mathrm{d} t+g(t, x) \mathrm{d} \beta,
$$

where $x$ is the system state, $f \in \mathscr{L}_{1}\left(\mathbb{R}_{+} ; \mathbb{R}^{n}\right)$ and $g \in \mathscr{L}_{2}\left(\mathbb{R}_{+} ; \mathbb{R}^{n \times m}\right)$ are known functions, and $\beta$ is the onedimensional standard Wiener process.

For $V(t, x) \in \mathscr{C}^{2,1}\left(\mathbb{R} ; \mathbb{R}^{n} \times \mathbb{R}_{+}\right)$, the infinitesimal generator along (4) is defined by

$$
\begin{aligned}
\mathscr{L} V(t, x)= & V_{t}(t, x)+V_{x}(t, x) f(t, x) \\
& +\frac{1}{2} \operatorname{Tr}\left[g^{T}(t, x) V_{x x}(t, x) g(t, x)\right],
\end{aligned}
$$

where $\operatorname{Tr}$ is the trace of a matrix, and

$$
\begin{aligned}
V_{t}(t, x) & =\frac{\partial V(t, x)}{\partial t} \\
V_{x}(t, x) & =\left(\frac{\partial V(t, x)}{\partial \vartheta_{1}}, \ldots, \frac{\partial V(t, x)}{\partial \vartheta_{n}}\right) \\
V_{x x}(t, x) & =\left(\frac{\partial^{2} V(t, x)}{\partial \vartheta_{i} \partial \vartheta_{j}}\right)_{n \times n} .
\end{aligned}
$$

Definition 1 (see [28]). Let $p>0$. System (4) is said to be asymptotically bounded in $p$-th moment if there is a positive constant $H$ such that

$$
\lim _{t \longrightarrow \infty} \sup E\left|x\left(t ; x_{0}\right)\right|^{p} \leq H,
$$

for all $\left(t_{0}, x_{0}\right) \in \mathbb{R}_{+} \times \mathbb{R}^{n}$. When $p=2$, we say system (4) is asymptotically bounded in mean square.
Lemma 1. For system (4), assume that there exists a function $V \in C^{2,1}\left(\mathbb{R}^{n} \times \mathbb{R}_{+}, \mathbb{R}_{+}\right)$, positive constants $k_{i}, k_{i}^{\prime}, p_{i}, p_{i}^{\prime}, c, d_{c}$, such that

$$
\begin{aligned}
& \sum_{i=1}^{n} k_{i}\left|x_{i}\right|^{p_{i}} \leq V(x(t), t) \leq \sum_{i=1}^{n} k_{i}^{\prime}\left|x_{i}\right|^{p_{i}^{\prime}}, \\
& \mathscr{L} V(x(t), t) \leq-\lambda V(x(t), t)+d_{c} .
\end{aligned}
$$

Then, there exists a unique strong solution $x(t)=x\left(t ; x_{0}, t_{0}\right)$ of system (4) for each $x\left(t_{0}\right)=x_{0} \in \mathbb{R}^{n}$, and system (4) is $p$-th moment exponentially practically stable, where $p=\min \left\{p_{1}, \ldots, p_{n}\right\}$.

Lemma 2. For any vectors $x, y \in \mathbb{R}^{n}$ and any scalars $\epsilon>0, p>1$, there holds $x^{T} y \leq\left(\epsilon^{p} / p\right)|x|^{p}+\left(1 / q \epsilon^{q}\right)|y|^{q}$, where $q=(p /(p-1))$.

\section{Position Loop Control}

In this section, the DOBC method and backsetpping method are used to construct the flight controller for helicopters under stochastic disturbances. We first build disturbance observers to estimate the common disturbances $\delta_{1}$ and $\delta_{2}$.

3.1. Disturbance Observer Designed for Position Control. In what follows, the disturbance observer is designed to estimate the disturbance $\delta_{1}$,

$$
\begin{aligned}
\widehat{\delta}_{1} & =\sigma_{1}+\rho_{1}(v), \\
\mathrm{d} \sigma_{1} & =-\frac{\partial \rho_{1}(v)}{\partial v}\left(\left(-g e_{3}+\frac{1}{m} R f\right) \mathrm{d} t+\widehat{\delta}_{1} \mathrm{~d} t\right)+\sigma_{11} \mathrm{~d} t,
\end{aligned}
$$

where $\sigma_{1}$ is an auxiliary variable, $\sigma_{11}$ is an auxiliary function to be designed, and $\rho_{1}(v)$ is a nonlinear function to be designed.

Estimate error is defined as $\widetilde{\delta}_{1}=\widehat{\delta}_{1}-\delta_{1}$. Then, the dynamic of the estimate error is given by

$$
\mathrm{d} \widetilde{\delta}_{1}=-\frac{\partial \rho_{1}(v)}{\partial v}\left(\widetilde{\delta}_{1} \mathrm{~d} t+G_{1} \mathrm{~d} \beta_{1}\right)-\dot{\delta}_{1} \mathrm{~d} t+\sigma_{11} \mathrm{~d} t
$$

The disturbance observer gain is selected as $\left(\partial \rho_{1}(v) / \partial v\right)=L_{1}$, with $L_{1}=-\operatorname{diag}\left\{\begin{array}{llll}l_{1} & l_{1} & l_{1}\end{array}\right\}$, where $l_{1}>0$.

Choose the Lyapunov function as follows: 


$$
V_{01}=\frac{1}{4}\left(\widetilde{\delta}_{1}^{T} \widetilde{\delta}_{1}\right)^{2}
$$

Then, the infinitesimal generator of $V_{01}$ along with (11) is shown as follows:

$$
\mathscr{L} V_{01}=-L_{1}\left(\widetilde{\delta}_{1}^{T} \widetilde{\delta}_{1}\right)^{2}-\widetilde{\delta}_{1}^{T} \widetilde{\delta}_{1} \widetilde{\delta}_{1}^{T} \dot{\delta}_{1}+\widetilde{\delta}_{1}^{T} \widetilde{\delta}_{1} \widetilde{\delta}_{1}^{T} \sigma_{11}+\frac{1}{2} \operatorname{Tr}\left(G_{1}^{T} L_{1}\left(2 \widetilde{\delta}_{1} \widetilde{\delta}_{1}^{T}+\widetilde{\delta}_{1}^{T} \widetilde{\delta}_{1} I\right) L_{1} G_{1}\right)
$$

From Lemma 2, there exist parameters $\varepsilon_{01}>0$ and $\varepsilon_{02}>0$ such that

$$
\begin{gathered}
-\widetilde{\delta}_{1}^{T} \widetilde{\delta}_{1} \widetilde{\delta}_{1}^{T} \dot{\delta}_{1} \leq \frac{3 \varepsilon_{01}^{(4 / 3)}}{4}\left(\widetilde{\delta}_{1}^{T} \widetilde{\delta}_{1}\right)^{2}+\frac{1}{4 \varepsilon_{01}^{4}}\left\|\dot{\delta}_{1}\right\|^{4}, \\
\frac{1}{2} \operatorname{Tr}\left(G_{1}^{T} L_{1}\left(2 \widetilde{\delta}_{1} \widetilde{\delta}_{1}^{T}+\widetilde{\delta}_{1}^{T} \widetilde{\delta}_{1} I\right) L_{1} G_{1}\right) \leq \frac{3}{2}\left\|\widetilde{\delta}_{1}\right\|^{2}\left\|L_{1} G_{1}\right\|_{F}^{2} \leq \frac{3 \varepsilon_{02}^{2}}{4}\left\|L_{1} G_{1}\right\|_{F}^{4}\left(\widetilde{\delta}_{1}^{T} \widetilde{\delta}_{1}\right)^{2}+\frac{3}{4 \varepsilon_{02}^{2}} . \\
\mathrm{d} p=v \mathrm{~d} t,
\end{gathered}
$$

Design $\sigma_{11}$ as

$$
\sigma_{11}=-\left(\frac{3 \varepsilon_{01}^{(4 / 3)}}{4}+\frac{3 \varepsilon_{02}^{2}}{4}\left\|L_{1} G_{1}\right\|_{F}^{4}\right) \tilde{\delta}_{1}+\sigma_{12},
$$

where $\sigma_{12}$ is introduced in the following steps. Hence, from (15) and $\left\|\dot{\delta}_{1}\right\| \leq \mu_{1}$, we have

$$
\begin{aligned}
\mathscr{L} V_{01} & \leq-L_{1}\left(\widetilde{\delta}_{1}^{T} \widetilde{\delta}_{1}\right)^{2}+\frac{1}{4 \varepsilon_{01}^{4}}\left\|\mu_{1}\right\|^{4}+\frac{3}{4 \varepsilon_{02}^{2}}+\widetilde{\delta}_{1}^{T} \widetilde{\delta}_{1} \widetilde{\delta}_{1}^{T} \sigma_{12} \\
& =-L_{1}\left(\widetilde{\delta}_{1}^{T} \widetilde{\delta}_{1}\right)^{2}+\widetilde{\delta}_{1}^{T} \widetilde{\delta}_{1} \widetilde{\delta}_{1}^{T} \sigma_{12}+H_{01},
\end{aligned}
$$

where

$$
H_{01}=\frac{1}{4 \varepsilon_{01}^{4}}\left\|\mu_{1}\right\|^{4}+\frac{3}{4 \varepsilon_{02}^{2}} .
$$

3.2. Position Loop Controller Designed. Consider the position loop system as follows:

$$
\mathrm{d} v=\left(-g e_{3}+\frac{1}{m} R f\right) \mathrm{d} t+\delta_{1} \mathrm{~d} t+G_{1} \mathrm{~d} \beta_{1} .
$$

The predefined position is $p_{r}=\left(x_{r}, y_{r}, z_{r}\right)$, define the tracking error as $e_{p}=p-p_{r}$. The dynamic of the error system is given by

$$
\begin{aligned}
& \mathrm{d} e_{p}=v \mathrm{~d} t-\mathrm{d} p_{r}=e_{v} \mathrm{~d} t \\
& \mathrm{~d} e_{v}=\left(-g e_{3}+\frac{T_{m}}{m} R_{3 e}+\frac{T_{m}}{m} R_{3 c}-\ddot{p}_{r}+\widetilde{\delta}_{1}+\widehat{\delta}_{1}\right) \mathrm{d} t+G_{1} \mathrm{~d} \beta_{1} .
\end{aligned}
$$

Define the controller $u_{1}=\left(T_{m} / m\right) R_{3 c} \quad$ and $R_{3 c}=\left(\begin{array}{lll}R_{13 c} & R_{23 c} & R_{33 c}\end{array}\right)^{T}$, and

$$
u_{1}=g e_{3}+\ddot{p}_{r}-\widehat{\delta}_{1}-k_{1} \tanh \left(n_{1} e_{p}+n_{2} e_{v}\right)-k_{2} \tanh \left(n_{2} e_{v}\right) \text {, }
$$

where $k_{1}>0, k_{2}>0, n_{1}>0$, and $n_{2}>0$ are parameters to be designed. At this point, $R_{3 c}$ and $T_{m}$ can be constructed as

$$
\begin{aligned}
& R_{3 c}=\frac{m}{T_{m}} u_{1}, \\
& T_{m}=m\left\|g e_{3}+\ddot{p}_{r}-\widehat{\delta}_{1}-k_{1} \tanh \left(n_{1} e_{p}+n_{2} e_{v}\right)-k_{2} \tanh \left(n_{2} e_{v}\right)\right\| .
\end{aligned}
$$

Then, the closed-loop position loop system is written as

$$
\begin{aligned}
& \mathrm{d} e_{p}=e_{v} \mathrm{~d} t \\
& \mathrm{~d} e_{v}=\left(-k_{1} \tanh \left(n_{1} e_{p}+n_{2} e_{v}\right)-k_{2} \tanh \left(n_{2} e_{v}\right)+\frac{T_{m}}{m} R_{3 e}+\widetilde{\delta}_{1}\right) \mathrm{d} t+G_{1} \mathrm{~d} \beta_{1} .
\end{aligned}
$$


Remark 1. In fact, the helicopter should not be overturn under controller (21), for the continuity of $\cos \phi \cos \theta$. So that, we guarantee $\cos \phi \cos \theta>0$ to ensure that the helicopter will not be overturn. Meanwhile, the defined signal $R_{33 c}$ should also satisfy $R_{33 c}>0$. Thus, the parameters $k_{1}$ and $k_{2}$ are designed to satisfy $g+\ddot{z}_{r}+\widehat{\delta}_{1} e-k_{1}-k_{2}>0$. In fact, the acceleration of disturbance in $z$ axis is far less than acceleration of gravity.

Consider $\left\|R_{3}\right\|=1, R_{3 e}=R_{3}-R_{3 c}, \bar{R}_{3}=\left(\begin{array}{l}R_{13} \\ R_{23}\end{array}\right)$, and $\bar{R}_{3 c}=\left(\begin{array}{l}R_{13 c} \\ R_{23 c}\end{array}\right)$. Thus,

$$
\begin{aligned}
R_{3} & =\left(\begin{array}{l}
R_{13} \\
R_{23} \\
R_{33}
\end{array}\right)=\left(\begin{array}{c}
R_{13} \\
R_{23} \\
\sqrt{1-R_{13}^{2}-R_{23}^{2}}
\end{array}\right), \\
R_{3 e} & =\left(\begin{array}{l}
R_{13} \\
R_{23} \\
R_{33}
\end{array}\right)-\left(\begin{array}{c}
R_{13 c} \\
R_{23 c} \\
R_{33 c}
\end{array}\right)=\left(\begin{array}{c}
R_{13 e} \\
R_{23 e} \\
\sqrt{1-R_{13}^{2}-R_{23}^{2}}-\sqrt{1-R_{13 c}^{2}-R_{23 c}^{2}}
\end{array}\right) .
\end{aligned}
$$

Due to $R_{33}>0$ and $R_{33 c}>0$, we have

$$
\sqrt{1-R_{13}^{2}-R_{23}^{2}}-\sqrt{1-R_{13 c}^{2}-R_{23 c}^{2}}=\frac{\alpha_{1} \bar{R}_{3 e}}{\sqrt{1-R_{13}^{2}-R_{23}^{2}}+\sqrt{1-R_{13 c}^{2}-R_{23 c}^{2}}}
$$

where $\alpha_{1}=-\left(R_{13}+R_{13 c}, R_{23}+R_{23 c}\right)$. Hence,

$$
R_{3 e}=\left(\begin{array}{c}
\bar{R}_{3 e} \\
\alpha_{1} \bar{R}_{3 e}
\end{array}\right)=\left(\begin{array}{c}
I_{2} \\
\frac{\alpha_{1}}{\sqrt{1-R_{13}^{2}-R_{23}^{2}}+\sqrt{1-R_{13 c}^{2}-R_{23 c}^{2}}}
\end{array}\right) \bar{R}_{3 e}=\alpha \bar{R}_{3 e}=\alpha\left(\bar{R}_{3}-\bar{R}_{3 c}\right)
$$

where $\alpha=\left(\left(\alpha_{1} /\left({\sqrt{1-R_{13}^{2}-R_{23}^{2}}}_{2}+\sqrt{1-R_{13 c}^{2}-R_{23 c}^{2}}\right)\right)\right.$

The velocity tracking error equation (23) can be rewritten as

$$
\mathrm{d} e_{v}=\left(-k_{1} \tanh \left(n_{1} e_{p}+n_{2} e_{v}\right)-k_{2} \tanh \left(n_{2} e_{v}\right)+\frac{T_{m}}{m} \alpha \bar{R}_{3 e}+\widetilde{\delta}_{1}\right) \mathrm{d} t+G_{1} \mathrm{~d} \beta_{1}
$$

Choose Lyapunov function $V_{11}(t)$ as follows:

$$
V_{11}(t)=k_{1} \int_{0}^{n_{1} e_{p}+n_{2} e_{v}} \tanh (\mu) \mathrm{d} \mu+k_{2} \int_{0}^{n_{2} e_{v}} \tanh (\mu) \mathrm{d} \mu+\frac{n_{1}}{2} e_{v}^{T} e_{v} .
$$

Then, the infinitesimal generator of $V_{11}$ is represented as 


$$
\begin{aligned}
\mathscr{L} V_{11}(t)= & -n_{2}\left(k_{1} \tanh \left(n_{1} e_{p}+n_{2} e_{v}\right)+k_{2} \tanh \left(n_{2} e_{v}\right)\right)^{T} \\
& \times\left(k_{1} \tanh \left(n_{1} e_{p}+n_{2} e_{v}\right)+k_{2} \tanh \left(n_{2} e_{v}\right)\right)-n_{1} k_{2} e_{v}^{T} \tanh \left(n_{2} e_{v}\right) \\
& +F(t)^{T}\left(\frac{T_{m}}{m} \alpha \bar{R}_{3 e}+\widetilde{\delta}_{1}\right)+\frac{1}{2} \operatorname{Tr}\left(\left(\begin{array}{c}
0 \\
G_{1}
\end{array}\right)^{T}\left(\begin{array}{c}
\frac{\partial^{2} V_{11}}{\partial e_{p}^{2}} \frac{\partial^{2} V_{11}}{\partial e_{p} \partial e_{v}} \\
\frac{\partial^{2} V_{11}}{\partial e_{p} \partial e_{v}} \frac{\partial^{2} V_{11}}{\partial e_{v}^{2}}
\end{array}\right)\left(\begin{array}{c}
0 \\
G_{1}
\end{array}\right)\right)
\end{aligned}
$$

where

$$
\begin{aligned}
F(t)= & n_{1} e_{v}+n_{2} k_{1} \tanh \left(n_{1} e_{p}+n_{2} e_{v}\right)+n_{2} k_{2} \tanh \left(n_{2} e_{v}\right), \\
\frac{\partial^{2} V_{11}}{\partial e_{p}^{2}}= & \operatorname{diag}\left\{n_{1}^{2} k_{1}\left(1+\tanh ^{2}\left(n_{1} e_{p_{1}}+n_{2} e_{v_{1}}\right)\right), n_{1}^{2} k_{1}\left(1+\tanh ^{2}\left(n_{1} e_{p_{2}}+n_{2} e_{v_{2}}\right)\right), n_{1}^{2} k_{1}\left(1+\tanh ^{2}\left(n_{1} e_{p_{3}}+n_{2} e_{v_{3}}\right)\right)\right\}, \\
\frac{\partial^{2} V_{11}}{\partial e_{p} \partial e_{v}}= & \operatorname{diag}\left\{n_{1} n_{2} k_{1}\left(1+\tanh ^{2}\left(n_{1} e_{p_{1}}+n_{2} e_{v_{1}}\right)\right), n_{1} n_{2} k_{1}\left(1+\tanh ^{2}\left(n_{1} e_{p_{2}}+n_{2} e_{v_{2}}\right)\right), n_{1} n_{2} k_{1}\left(1+\tanh ^{2}\left(n_{1} e_{p_{3}}+n_{2} e_{v_{3}}\right)\right)\right\}, \\
\frac{\partial^{2} V_{11}}{\partial e_{v}^{2}}= & \operatorname{diag}\left\{n_{2}^{2} k_{1}\left(1+\tanh ^{2}\left(n_{1} e_{p_{1}}+n_{2} e_{v_{1}}\right)\right)+n_{2}^{2} k_{2}\left(1+\tanh ^{2}\left(n_{2} e_{v_{1}}\right)\right),\right. \\
& n_{2}^{2} k_{1}\left(1+\tanh ^{2}\left(n_{1} e_{p_{2}}+n_{2} e_{v_{2}}\right)\right)+n_{2}^{2} k_{2}\left(1+\tanh ^{2}\left(n_{2} e_{v_{2}}\right)\right), \\
& \left.n_{2}^{2} k_{1}\left(1+\tanh ^{2}\left(n_{1} e_{p_{3}}+n_{2} e_{v_{3}}\right)\right)+n_{2}^{2} k_{2}\left(1+\tanh ^{2}\left(n_{2} e_{v_{3}}\right)\right)\right\}+\operatorname{diag}\{1,1,1\} .
\end{aligned}
$$

For the function $\|\tanh (\cdot)\| \leq 1$ and the bounded parameter $G_{1}$, there exists a parameter $H_{11}>0$, such that

$$
\frac{1}{2} \operatorname{Tr}\left(\left(\begin{array}{c}
0 \\
G_{1}
\end{array}\right)^{T}\left(\begin{array}{cc}
\frac{\partial^{2} V_{11}}{\partial e_{p}^{2}} & \frac{\partial^{2} V_{11}}{\partial e_{p} \partial e_{v}} \\
\frac{\partial^{2} V_{11}}{\partial e_{p} \partial e_{v}} & \frac{\partial^{2} V_{11}}{\partial e_{v}^{2}}
\end{array}\right)\left(\begin{array}{c}
0 \\
G_{1}
\end{array}\right)\right) \leq H_{11} .
$$

$$
\begin{aligned}
\mathscr{L} V_{11}(t) \leq & -n_{2}\left(k_{1} \tanh \left(n_{1} e_{p}+n_{2} e_{v}\right)+k_{2} \tanh \left(n_{2} e_{v}\right)\right)^{T}\left(k_{1} \tanh \left(n_{1} e_{p}+n_{2} e_{v}\right)+k_{2} \tanh \left(n_{2} e_{v}\right)\right) \\
& -\frac{n_{1}}{n_{2}} k_{2} \tanh \left(n_{2} e_{v}\right)^{T} \tanh \left(n_{2} e_{v}\right)+F(t)^{T}\left(\frac{T_{m}}{m} \alpha \bar{R}_{3 e}+\widetilde{\delta}_{1}\right)+H_{11} \\
= & -\left(\begin{array}{cc}
\tanh \left(n_{1} e_{p}+n_{2} e_{v}\right) \\
\tanh \left(n_{2} e_{v}\right)
\end{array}\right)\left(\begin{array}{cc}
n_{2} k_{1}^{2} & n_{2} k_{1} k_{2} \\
n_{2} k_{1} k_{2} & n_{2} k_{2}^{2}+\frac{n_{1}}{n_{2}} k_{2}
\end{array}\right)\left(\begin{array}{c}
\tanh \left(n_{1} e_{p}+n_{2} e_{v}\right) \\
\tanh \left(n_{2} e_{v}\right)
\end{array}\right) \\
& +F(t)^{T} \widetilde{\delta}_{1}+F(t)^{T} \frac{T_{m}}{m} \alpha \bar{R}_{3 e}+H_{11} .
\end{aligned}
$$


In fact, according to Lemma 2, there exist parameters $\varepsilon_{11}>0$ and $\varepsilon_{12}>0$, such that

$$
\begin{gathered}
F(t)^{T} \widetilde{\delta}_{1} \leq \frac{\varepsilon_{11}}{3} \widetilde{\delta}_{1}^{T} \widetilde{\delta}_{1} \widetilde{\delta}_{1}^{T}\|F(t)\|^{2} F(t)+\frac{2}{3 \varepsilon_{11}^{(1 / 2)}} \\
F(t)^{T} \frac{T_{m}}{m} \alpha \bar{R}_{3 e} \leq \frac{\varepsilon_{12} T_{m}^{3}\|\alpha\|^{2}\|F(t)\|^{2}}{3 m^{3}} \bar{R}_{3 e}^{T} \bar{R}_{3 e} \bar{R}_{3 e}^{T} \alpha^{T} F(t)+\frac{2}{3 \varepsilon_{12}^{(1 / 2)}} .
\end{gathered}
$$

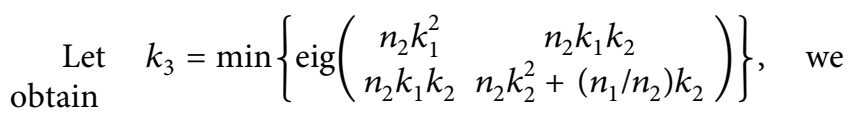

$$
\begin{aligned}
\mathscr{L} V_{11}(t) \leq & -k_{3}\left(\begin{array}{c}
\tanh \left(n_{1} e_{p}+n_{2} e_{v}\right) \\
\tanh \left(n_{2} e_{v}\right)
\end{array}\right)\left(\begin{array}{c}
\tanh \left(n_{1} e_{p}+n_{2} e_{v}\right) \\
\tanh \left(n_{2} e_{v}\right)
\end{array}\right)+\frac{\varepsilon_{12} T_{m}^{3}\|\alpha\|^{2}\|F(t)\|^{2}}{3 m^{3}} \bar{R}_{3 e}^{T} \bar{R}_{3 e} \bar{R}_{3 e}^{T} \alpha^{T} F(t) \\
& +\frac{\varepsilon_{11}}{3} \widetilde{\delta}_{1}^{T} \widetilde{\delta}_{1} \widetilde{\delta}_{1}^{T}\|F(t)\|^{2} F(t)+\frac{2}{3 \varepsilon_{11}^{(1 / 2)}}+\frac{2}{3 \varepsilon_{12}^{(1 / 2)}}+H_{11} .
\end{aligned}
$$

Since $h(x)=(\tanh (x) / x)$ is a continuous function, for any $\bar{x}>0,0 \leq x \leq \bar{x}$, there exists a positive number $\beta(\bar{x})$ such that $\beta\|x\| \leq\|\tanh (x)\| \leq\|x\|$. Thus,

$$
\begin{aligned}
-\tanh \left(n_{1} e_{p}+\right. & \left.n_{2} e_{v}\right)^{T} \tanh \left(n_{1} e_{p}+n_{2} e_{v}\right) \leq-\beta^{2}\left(n_{1} e_{p}+n_{2} e_{v}\right)^{T}\left(n_{1} e_{p}+n_{2} e_{v}\right), \\
- & \tanh \left(n_{2} e_{v}\right)^{T} \tanh \left(n_{2} e_{v}\right) \leq-\beta^{2} n_{2}^{2} e_{v}^{T} e_{v} .
\end{aligned}
$$

Define Lyapunov function as follows:

Based on (16) and (34), we obtain

$$
V_{1}(t)=V_{01}(t)+V_{11}(t) .
$$

$$
\begin{aligned}
\mathscr{L} V_{1}(t) \leq & -k_{3} \beta^{2}\left(n_{1} e_{p}+n_{2} e_{v}\right)^{T}\left(n_{1} e_{p}+n_{2} e_{v}\right)-k_{3} \beta^{2} n_{2}^{2} e_{v}^{T} e_{v} \\
& +\frac{\varepsilon_{12} T_{m}^{3}\|\alpha\|^{2}\|F(t)\|^{2}}{3 m^{3}} \bar{R}_{3 e}^{T} \bar{R}_{3 e} \bar{R}_{3 e}^{T} \alpha^{T} F(t)+\frac{\varepsilon_{11}}{3} \widetilde{\delta}_{1}^{T} \widetilde{\delta}_{1} \widetilde{\delta}_{1}^{T}\|F(t)\|^{2} F(t) \\
& +\frac{2}{3 \varepsilon_{11}^{(1 / 2)}}+\frac{2}{3 \varepsilon_{12}^{(1 / 2)}}+H_{11}-L_{1}\left(\widetilde{\delta}_{1}^{T} \widetilde{\delta}_{1}\right)^{2}+\widetilde{\delta}_{1}^{T} \widetilde{\delta}_{1} \widetilde{\delta}_{1}^{T} \sigma_{12}+H_{01} .
\end{aligned}
$$

Hence, $\sigma_{12}$ is designed as

$$
\sigma_{12}=-\frac{\varepsilon_{11}}{3}\|F(t)\|^{2} F(t) .
$$

Then, 


$$
\mathscr{L} V_{1}(t) \leq-l_{1}\left(\widetilde{\delta}_{1}^{T} \widetilde{\delta}_{1}\right)^{2}-k_{4} e_{p}^{T} e_{p}-k_{4} e_{v}^{T} e_{v}+\frac{\varepsilon_{12} T_{m}^{3}\|\alpha\|^{2}\|F(t)\|^{2}}{3 m^{3}} \bar{R}_{3 e}^{T} \bar{R}_{3 e} \bar{R}_{3 e}^{T} \alpha^{T} F(t)+H_{1},
$$

where $k_{4}=\min \left\{\operatorname{eig}\left\{\left(\begin{array}{cc}k_{3} \beta^{2} n_{1}^{2} & k_{3} \beta^{2} n_{1} n_{2} \\ k_{3} \beta^{2} n_{1} n_{2} & 2 k_{3} \beta^{2} n_{2}^{2}\end{array}\right)\right\}\right\}>0 \quad$ and $H_{1}=H_{11}+H_{01}+\left(2 / 3 \varepsilon_{11}^{(1 / 2)}\right)+\left(2 / 3 \varepsilon_{12}^{(1 / 2)}\right)$.
Moreover, the error dynamic of disturbance $\widetilde{\delta}_{1}$ can be rewritten as

$$
\mathrm{d} \widetilde{\delta}_{1}=-\left(L_{1}+\left(\frac{3 \varepsilon_{01}^{(4 / 3)}}{4}+\frac{3 \varepsilon_{02}^{2}}{4}\left\|L_{1} G_{1}\right\|_{F}^{4}\right)\right) \widetilde{\delta}_{1} \mathrm{~d} t-L_{1} G_{1} \mathrm{~d} \beta_{1}-\dot{\delta}_{1} \mathrm{~d} t-\frac{\varepsilon_{11}}{3}\|F(t)\|^{2} F(t) \mathrm{d} t .
$$

\section{Attitude Loop Control}

In this section, the DOBC method and backsetpping method are used to construct the flight controller for helicopters under stochastic disturbances. We first build disturbance observers to estimate the common disturbances $\delta_{1}$ and $\delta_{2}$.

4.1. Disturbance Observer Designed for Attitude Control. In order to estimate the disturbance $\delta_{2}$, we design the following disturbance observers:

$$
\begin{aligned}
\widehat{\delta}_{2} & =\sigma_{2}+J \rho_{2}(w), \\
\mathrm{d} \sigma_{2} & =-\frac{\partial \rho_{2}(w)}{\partial w}\left(\left(-w^{\times} J w+\tau\right) \mathrm{d} t+\widehat{\delta}_{2} \mathrm{~d} t\right)+\sigma_{02} \mathrm{~d} t,
\end{aligned}
$$

where $\sigma_{2}$ is an auxiliary variable, $\sigma_{02}$ is an auxiliary function to be designed, and $\rho_{2}(v)$ is a nonlinear function to be designed.

Define estimate error as $\widetilde{\delta}_{2}=\widehat{\delta}_{2}-\delta_{2}$. Then, the dynamic of the estimate error can be shown as

$$
\mathrm{d} \widetilde{\delta}_{2}=-\frac{\partial \rho_{2}(w)}{\partial w}\left(\widetilde{\delta}_{2} \mathrm{~d} t+G_{2} \mathrm{~d} \beta_{2}\right)-\dot{\delta}_{2} \mathrm{~d} t+\sigma_{02} \mathrm{~d} t
$$

The disturbance observer gain is selected as $\left(\partial \rho_{2}(w) / \partial w\right)=L_{2}$, with $L_{2}=-\operatorname{diag}\left\{l_{2} l_{2} l_{2}\right\}$, where $l_{2}>0$.

Choose Lyapunov function as follows:

$$
V_{02}=\frac{1}{4}\left(\widetilde{\delta}_{2}^{T} \widetilde{\delta}_{2}\right)^{2}
$$

Similarly with $V_{01}$, for $V_{02}$, there exist $\varepsilon_{03}>0$ and $\varepsilon_{04}>0$, such that

$$
\mathscr{L} V_{02} \leq-l_{2}\left(\widetilde{\delta}_{2}^{T} \widetilde{\delta}_{2}\right)^{2}+H_{02}
$$

where

$$
H_{02}=\frac{1}{4 \varepsilon_{03}^{4}}\left\|\mu_{2}\right\|^{4}+\frac{3}{4 \varepsilon_{04}^{2}} .
$$

Design the auxiliary variable $\sigma_{02}$ as follows:

$$
\sigma_{02}=-\left(\frac{3 \varepsilon_{03}^{(4 / 3)}}{4}+\frac{3 \varepsilon_{04}^{2}}{4}\left\|L_{2} G_{2}\right\|_{F}^{4}\right) \widetilde{\delta}_{2} .
$$

The error dynamics of disturbance (42) can be rewritten as

$$
\mathrm{d} \widetilde{\delta}_{2}=-\left(l_{2}+\frac{3 \varepsilon_{03}^{(4 / 3)}}{4}+\frac{3 \varepsilon_{04}^{2}}{4}\left\|L_{2} G_{2}\right\|_{F}^{4}\right) \widetilde{\delta}_{2} \mathrm{~d} t-\dot{\delta}_{2} \mathrm{~d} t-L_{2} G_{2} \mathrm{~d} \beta_{2} .
$$

4.2. Attitude Loop Controller Designed. The predefined tracking signals are position and yaw angle, which are denoted by $p_{r}$ and $\psi_{r}$. After designing the position loop control, the reasonable attitude tracking signals are obtained, which are given as $\bar{R}_{3 c}$ and $\psi_{r}$. Moreover, defining $\gamma_{r}=\left(\begin{array}{ll}R_{3}^{T} \psi\end{array}\right)$ and $\gamma=\left(\begin{array}{lll}\theta \phi & \psi\end{array}\right)$, we can confirm that $\left\|\partial \gamma_{r} / \partial \gamma\right\|=\cos \theta \neq 0$, for $|\theta|<(\pi / 2)$. Hence, the mapping that from $\gamma$ to $\gamma_{r}$ is a local topological homeomorphism, which means that the dynamic of $\gamma_{r}$ can represent the dynamic of $\gamma$ for $|\theta|<(\pi / 2)$. The error dynamic of $\gamma_{r}$ is given by

$$
\begin{aligned}
\mathrm{d} \bar{R}_{3 e} & =\widehat{R}_{3} w_{1} \mathrm{~d} t-\mathrm{d} \bar{R}_{3 c}, \\
\mathrm{~d} e_{\psi} & =\left(\frac{\sin \phi}{\cos \theta} q+\frac{\cos \phi}{\cos \theta} r\right) \mathrm{d} t-\mathrm{d} \psi_{r},
\end{aligned}
$$

where $w_{1}=\left(\begin{array}{ll}p & q\end{array}\right)^{T}$ and $\widehat{R}_{3}=\left(\begin{array}{ll}-R_{12} & R_{11} \\ -R_{22} & R_{21}\end{array}\right)$.

For (48), $\mathrm{d} R_{3 c}$ can be written as

$$
\mathrm{d} R_{3 c}=\mathrm{d}\left(\frac{u_{1}}{\sqrt{u_{1}^{T} u_{1}}}\right)=\frac{1}{\sqrt{u_{1}^{T} u_{1}}}\left(I_{3}-\frac{u_{1} u_{1}^{T}}{u_{1}^{T} u_{1}}\right) \mathrm{d} u_{1} .
$$

For $\mathrm{d} u_{1}$, we have

$$
\mathrm{d} u_{1}=\mathrm{d} \ddot{p}_{r}-\mathrm{d} \widehat{\delta}_{1}-k_{1} \mathrm{~d} \tanh \left(n_{1} e_{p}+n_{2} e_{v}\right)-k_{2} \mathrm{dtanh}\left(n_{2} e_{v}\right) .
$$

From (10), $\mathrm{d} \widehat{\delta}_{1}$ is written as follows:

$$
\mathrm{d} \widehat{\delta}_{1}=-\bar{l}_{1} \widetilde{\delta}_{1} \mathrm{~d} t-L_{1} G_{1} \mathrm{~d} \beta_{1}-\frac{\varepsilon_{11}}{3}\|F(t)\|^{2} F(t) \mathrm{d} t,
$$

where $\bar{l}_{1}=l_{1}+\left(3 \varepsilon_{01}^{(4 / 3)} / 4\right)+\left(3 \varepsilon_{02}^{2} / 4\right)\left\|L_{1} G_{1}\right\|_{F}^{4}$.

Hence, we get

$$
\mathrm{d} u_{1}=\Delta_{01}(t) \mathrm{d} t+\Delta_{02}(t) \widetilde{\delta}_{1} \mathrm{~d} t+\Delta_{03}(t) \mathrm{d} \beta_{1},
$$


for $x=\left(x_{1}, x_{2}, x_{3}\right), \quad \operatorname{Tanh}^{2}(x)=\operatorname{diag}\left\{\tanh ^{2}\left(x_{1}\right), \tanh ^{2}\right.$ $\left.\left(x_{2}\right) y, \tanh ^{2}\left(x_{3}\right)\right\}$, where

$$
\begin{aligned}
\Delta_{01}(t)= & \ddot{p}_{r}-k_{1} n_{1}\left(I+\operatorname{Tanh}^{2}\left(n_{1} e_{p}+n_{2} e_{v}\right)\right) e_{v}-\left(\left(k_{2} n_{2}\left(I+\operatorname{Tanh}^{2}\left(n_{2} e_{v}\right)\right)+k_{1} n_{2}\left(I+\operatorname{Tanh}^{2}\left(n_{1} e_{p}+n_{2} e_{v}\right)\right)\right)\right. \\
& \cdot\left(-k_{1} \tanh \left(n_{1} e_{p}+n_{2} e_{v}\right)-k_{2} \tanh \left(n_{2} e_{v}\right)+\frac{T_{m}}{m} \alpha \bar{R}_{3 e}\right)+\frac{\varepsilon_{11}}{3}\|F(t)\|^{2} F(t), \\
\Delta_{02}(t)= & \bar{l}_{1} I-\left(k_{2} n_{2}\left(I+\operatorname{Tanh}^{2}\left(n_{2} e_{v}\right)\right)+k_{1} n_{2}\left(I+\operatorname{Tanh}^{2}\left(n_{1} e_{p}+n_{2} e_{v}\right)\right)\right), \\
\Delta_{03}(t)= & L_{1} G_{1}-\left(k_{2} n_{2}\left(I+\operatorname{Tanh}^{2}\left(n_{2} e_{v}\right)\right)+k_{1} n_{2}\left(I+\operatorname{Tanh}^{2}\left(n_{1} e_{p}+n_{2} e_{v}\right)\right)\right) G_{1} .
\end{aligned}
$$

From (50),

$$
\begin{aligned}
\mathrm{d} R_{3 c}= & \frac{1}{\left\|u_{1}\right\|}\left(\Delta_{101}(t)+\Delta_{102}(t) e_{v}+\Delta_{103}(t) \Delta_{104}(t)+\frac{\varepsilon_{11}}{3}\|F(t)\|^{2} F(t)\right) \mathrm{d} t \\
& +\frac{1}{\left\|u_{1}\right\|} \Delta_{12}(t) \widetilde{\delta}_{1} \mathrm{~d} t+\frac{1}{\left\|u_{1}\right\|} \Delta_{13}(t) \mathrm{d} \beta_{1}
\end{aligned}
$$

where

$$
\begin{aligned}
& \Delta_{101}(t)=\left(I_{3}-\frac{u_{1} u_{1}^{T}}{u_{1}^{T} u_{1}}\right) \ddot{p}_{r}, \\
& \Delta_{102}(t)=-k_{2}\left(I_{3}-\frac{u_{1} u_{1}^{T}}{u_{1}^{T} u_{1}}\right)\left(I+\operatorname{Tanh}^{2}\left(e_{v}\right)\right), \\
& \Delta_{103}(t)=-\left(I_{3}-\frac{u_{1} u_{1}^{T}}{u_{1}^{T} u_{1}}\right)\left(k_{2}\left(I+\operatorname{Tanh}^{2}\left(e_{v}\right)\right)+k_{1}\left(I+\operatorname{Tanh}^{2}\left(e_{p}+e_{v}\right)\right)\right), \\
& \Delta_{104}(t)=\left(-k_{1} \tanh \left(e_{p}+e_{v}\right)-k_{2} \tanh \left(e_{v}\right)+\frac{T_{m}}{m} \alpha \bar{R}_{3 e}\right), \\
& \Delta_{12}(t)=\left(I_{3}-\frac{u_{1} u_{1}^{T}}{u_{1}^{T} u_{1}}\right)\left(\bar{l}_{1} I-\left(k_{2}\left(I+\operatorname{Tanh}^{2}\left(e_{v}\right)\right)+k_{1}\left(I+\operatorname{Tanh}^{2}\left(e_{p}+e_{v}\right)\right)\right)\right), \\
& \Delta_{13}(t)=\left(I_{3}-\frac{u_{1} u_{1}^{T}}{u_{1}^{T} u_{1}}\right)\left(L_{1} G_{1}-\left(k_{2}\left(I+\operatorname{Tanh}^{2}\left(e_{v}\right)\right)+k_{1}\left(I+\operatorname{Tanh}^{2}\left(e_{p}+e_{v}\right)\right)\right) G_{1}\right) .
\end{aligned}
$$

For $\left\|u_{1} u_{1}^{T}\right\|_{F} \leq\left\|u_{1}\right\|_{F}\left\|u_{1}^{T}\right\|_{F}=\left\|u_{1}\right\|^{2}$, one has 


$$
\left\|\left(I_{3}-\frac{u_{1} u_{1}^{T}}{u_{1}^{T} u_{1}}\right)\right\|_{F} \leq\left\|I_{3}\right\|_{F}+\frac{\left\|u_{1} u_{1}^{T}\right\|_{F}}{\left\|u_{1}\right\|^{2}} \leq \sqrt{3}+1 .
$$

Then, we confirm that

$$
\begin{aligned}
\left\|\Delta_{101}(t)\right\| & \leq(\sqrt{3}+1)\left\|\ddot{p}_{r}\right\|, \\
\left\|\Delta_{102}(t)\right\|_{F} & \leq k_{2}(\sqrt{3}+1)\left\|I+\operatorname{Tanh}^{2}\left(e_{v}\right)\right\|_{F} \leq k_{2}(3 \sqrt{2}+\sqrt{6}), \\
\left\|\Delta_{103}(t)\right\|_{F} & \leq(\sqrt{3}+1)\left\|k_{2}\left(I+\operatorname{Tanh}^{2}\left(e_{v}\right)\right)+k_{1}\left(I+\operatorname{Tanh}^{2}\left(e_{p}+e_{v}\right)\right)\right\|_{F} \leq(6+2 \sqrt{3})\left(k_{1}+k_{2}\right), \\
\left\|\Delta_{12}(t)\right\| & \leq(\sqrt{3}+1)\left(\bar{l}_{1}+2 \sqrt{3}\left(k_{1}+k_{2}\right)\right), \\
\left\|\Delta_{13}(t)\right\| & \leq(\sqrt{3}+1)\left(\left\|L_{1} G_{1}\right\|+2 \sqrt{3}\left(k_{1}+k_{2}\right)\left\|G_{1}\right\|\right) .
\end{aligned}
$$

Thus, (48) can be rewritten as

$$
\begin{aligned}
\mathrm{d} \bar{R}_{3 e}= & \widehat{R}_{3} e_{w_{1}} \mathrm{~d} t+\widehat{R}_{3} w_{1 c} \mathrm{~d} t-E_{0} \frac{1}{\left\|u_{1}\right\|}\left(\Delta_{101}(t)+\Delta_{102}(t) e_{v}+\Delta_{103}(t) \Delta_{104}(t)+\frac{\varepsilon_{11}}{3}\|F(t)\|^{2} F(t)\right) \mathrm{d} t \\
& -E_{0} \frac{1}{\left\|u_{1}\right\|} \Delta_{12}(t) \widetilde{\delta}_{1} \mathrm{~d} t-E_{0} \frac{1}{\left\|u_{1}\right\|} \Delta_{13}(t) \mathrm{d} \beta_{1},
\end{aligned}
$$

where $E_{0}=\left(\begin{array}{lll}1 & 0 & 0 \\ 0 & 1 & 0\end{array}\right)$.

Choose Lyapunov function $V_{21}(t)$ as

The infinitesimal generator of $V_{21}$ along with (60) is shown as

$$
V_{21}(t)=\frac{1}{4}\left(\bar{R}_{3 e}^{T} \bar{R}_{3 e}\right)^{2} .
$$

$$
\begin{aligned}
\mathscr{L} V_{21}(t)= & \bar{R}_{3 e}^{T} \bar{R}_{3 e} \bar{R}_{3 e}^{T} \widehat{R}_{3} e_{w_{1}}+\bar{R}_{3 e}^{T} \bar{R}_{3 e} \bar{R}_{3 e}^{T} \widehat{R}_{3} w_{1 c}-\bar{R}_{3 e}^{T} \bar{R}_{3 e} \bar{R}_{3 e}^{T} E_{0} \frac{1}{\left\|u_{1}\right\|} \Delta_{101}(t) \\
& -\bar{R}_{3 e}^{T} \bar{R}_{3 e} \bar{R}_{3 e}^{T} E_{0} \frac{1}{\left\|u_{1}\right\|} \Delta_{102}(t) e_{v}-\bar{R}_{3 e}^{T} \bar{R}_{3 e} \bar{R}_{3 e}^{T} E_{0} \frac{1}{\left\|u_{1}\right\|^{103}} \Delta_{104}(t) \Delta_{104}(t) \\
& -\frac{\varepsilon_{11}}{3} \bar{R}_{3 e}^{T} \bar{R}_{3 e} \bar{R}_{3 e}^{T} E_{0} \frac{1}{\left\|u_{1}\right\|}\|F(t)\|^{2} F(t)-\bar{R}_{3 e}^{T} \bar{R}_{3 e} \bar{R}_{3 e}^{T} E_{0} \frac{1}{\left\|u_{1}\right\|} \Delta_{12}(t) \widetilde{\delta}_{1} \\
& +\frac{1}{2\left\|u_{1}\right\|^{2}} \operatorname{Tr}\left(\Delta_{13}^{T}(t) E_{0}^{T}\left(2 \bar{R}_{3 e} \bar{R}_{3 e}^{T}+\bar{R}_{3 e}^{T} \bar{R}_{3 e} I\right) E_{0} \Delta_{13}(t)\right) .
\end{aligned}
$$

From Lemma 2, there exist parameters $\varepsilon_{21}>0, \varepsilon_{22}>0$, $\varepsilon_{231}>0, \varepsilon_{232}>0, \varepsilon_{24}>0$, and $\varepsilon_{25}>0$, such that 
Mathematical Problems in Engineering

11

$$
\begin{aligned}
& \bar{R}_{3 e}^{T} \bar{R}_{3 e} \bar{R}_{3 e}^{T} \widehat{R}_{3} e_{w_{1}} \leq \frac{3}{4 \varepsilon_{21}^{(1 / 3)}}\left(\bar{R}_{3 e}^{T} \bar{R}_{3 e}\right)^{2}+\frac{\varepsilon_{21}}{4}\left\|\widehat{R}_{3}\right\|^{4}\left(e_{w_{1}}^{T} e_{w_{1}}\right)^{2} \\
& -\bar{R}_{3 e}^{T} \bar{R}_{3 e} \bar{R}_{3 e}^{T} E_{0} \frac{1}{\left\|u_{1}\right\|} \Delta_{101}(t) \leq \frac{3 \varepsilon_{22}^{(1 / 3)}}{4\left\|u_{1}\right\|^{(4 / 3)}}\left(\bar{R}_{3 e}^{T} \bar{R}_{3 e}\right)^{2}+\frac{1}{4 \varepsilon_{22}}\left\|E_{0}\right\|^{4}\left\|\Delta_{101}(t)\right\|^{4}, \\
& -\bar{R}_{3 e}^{T} \bar{R}_{3 e} \bar{R}_{3 e}^{T} E_{0} \frac{1}{\left\|u_{1}\right\|} \Delta_{102}(t) e_{v} \leq \frac{\varepsilon_{231}}{2}\left(\bar{R}_{3 e}^{T} \bar{R}_{3 e}\right)^{2}+\frac{\varepsilon_{232}}{2\left\|u_{1}\right\|^{4}}\left(\bar{R}_{3 e}^{T} \bar{R}_{3 e}\right)^{2}\left(e_{v}^{T} e_{v}\right)^{2}+\frac{\left\|E_{0}\right\|^{4}\left\|\Delta_{102}(t)\right\|^{4}}{8 \varepsilon_{232} \varepsilon_{231}}, \\
& -\bar{R}_{3 e}^{T} \bar{R}_{3 e} \bar{R}_{3 e}^{T} E_{0} \frac{1}{\left\|u_{1}\right\|} \Delta_{12}(t) \widetilde{\delta}_{1} \leq \frac{3 \varepsilon_{24}^{(1 / 3)}}{4\left\|u_{1}\right\|^{(4 / 3)}}\left(\bar{R}_{3 e}^{T} \bar{R}_{3 e}\right)^{2}+\frac{1}{4 \varepsilon_{24}}\left\|E_{0}\right\|_{F}^{4}\left\|\Delta_{12}(t)\right\|^{4}\left(\widetilde{\delta}_{1}^{T} \widetilde{\delta}_{1}\right)^{2}, \\
& \frac{1}{2\left\|u_{1}\right\|^{2}} \operatorname{Tr}\left(\Delta_{13}^{T}(t) E_{0}^{T}\left(2 \bar{R}_{3 e} \bar{R}_{3 e}^{T}+\bar{R}_{3 e}^{T} \bar{R}_{3 e} I\right) E_{0} \Delta_{13}(t)\right) \leq \frac{3 \varepsilon_{25}}{4\left\|u_{1}\right\|^{2}}\left(\bar{R}_{3 e}^{T} \bar{R}_{3 e}\right)^{2}+\frac{1}{2 \varepsilon_{25}}\left\|E_{0} \Delta_{13}(t)\right\|_{F}^{4} .
\end{aligned}
$$

Furthermore, for $T_{m}=m\left\|u_{1}\right\|$, there exist parameters $\varepsilon_{26}>0$ and $\varepsilon_{27}>0$, such that

$$
\begin{aligned}
- & \bar{R}_{3 e}^{T} \bar{R}_{3 e} \bar{R}_{3 e}^{T} E_{0} \frac{1}{\left\|u_{1}\right\|^{103}} \Delta_{104}(t) \\
\leq & \frac{3 \varepsilon_{26}^{(1 / 3)}}{4\left\|u_{1}\right\|^{(4 / 3)}}\left(\bar{R}_{3 e}^{T} \bar{R}_{3 e}\right)^{2}+\frac{1}{4 \varepsilon_{26}}\left\|E_{0}\right\|_{F}^{4}\left\|\Delta_{103}(t)\right\|^{4}\left(\left\|k_{1} \tanh \left(e_{p}+e_{v}\right)\right\|+k_{2}\left\|\tanh \left(e_{v}\right)\right\|\right)^{4} \\
& +\frac{3 \varepsilon_{27}^{(1 / 3)}}{4}\left\|E_{0}\right\|_{F}^{(4 / 3)}\left\|\Delta_{103}(t)\right\|_{F}^{(4 / 3)}\left(\bar{R}_{3 e}^{T} \bar{R}_{3 e}\right)^{2}+\frac{1}{4 \varepsilon_{27}}\|\alpha\|_{F}^{4}\left(\bar{R}_{3 e}^{T} \bar{R}_{3 e}\right)^{2} .
\end{aligned}
$$

Then, $\mathscr{L} V_{21}(t)$ satisfies

$$
\begin{aligned}
& \mathscr{L} V_{21}(t) \leq\left(H_{25}+\frac{H_{24}}{4\left\|u_{1}\right\|^{(4 / 3)}}+\frac{3 \varepsilon_{25}}{4\left\|u_{1}\right\|^{2}}+\frac{1}{4 \varepsilon_{27}}\|\alpha\|_{F}^{4}\right)\left(\bar{R}_{3 e}^{T} \bar{R}_{3 e}\right)^{2}+\bar{R}_{3 e}^{T} \bar{R}_{3 e} \bar{R}_{3 e}^{T} \widehat{R}_{3} w_{1 c}+H_{23}\left(\widetilde{\delta}_{1}^{T} \widetilde{\delta}_{1}\right)^{2} \\
& -\frac{\varepsilon_{11}}{3} \bar{R}_{3 e}^{T} \bar{R}_{3 e} \bar{R}_{3 e}^{T} E_{0} \frac{1}{\left\|u_{1}\right\|}\|F(t)\|^{2} F(t)+\frac{\varepsilon_{232}}{2\left\|u_{1}\right\|^{4}}\left(\bar{R}_{3 e}^{T} \bar{R}_{3 e}\right)^{2}\left(e_{v}^{T} e_{v}\right)^{2}+\frac{\varepsilon_{21}}{4}\left\|\widehat{R}_{3}\right\|^{4}\left(e_{w_{1}}^{T} e_{w_{1}}\right)^{2}+H_{21} \text {, }
\end{aligned}
$$

where 


$$
\begin{aligned}
H_{21}= & \frac{1}{2 \varepsilon_{25}}\left\|E_{0}\right\|_{F}^{4}\left((\sqrt{3}+1)\left(\left\|L_{1} G_{1}\right\|+2 \sqrt{3}\left(k_{1}+k_{2}\right)\left\|G_{1}\right\|\right)\right)_{F}^{4}+\frac{1}{4 \varepsilon_{22}}\left\|E_{0}\right\|_{F}^{4}\|(\sqrt{3}+1)\|^{4}\left\|\ddot{p}_{r}\right\|^{4} \\
& +\frac{1}{4 \varepsilon_{26}}\left\|E_{0}\right\|_{F}^{2}(6+2 \sqrt{3})\left(k_{1}+k_{2}\right)^{4}\left(k_{1}+k_{2}\right)^{4}+\frac{\left\|E_{0}\right\|^{4}\left\|k_{2}(3 \sqrt{2}+\sqrt{6})\right\|^{4}}{8 \varepsilon_{232} \varepsilon_{231}} \\
H_{22}= & \frac{1}{4 \varepsilon_{23}}\left\|E_{0}\right\|_{F}^{4}\left(k_{2}(3 \sqrt{2}+\sqrt{6})\right)^{2} \\
H_{23}= & \frac{1}{4 \varepsilon_{24}}\left((\sqrt{3}+1)\left(\left\|\bar{l}_{1}\right\|_{F}+2 \sqrt{3}\left(k_{1}+k_{2}\right)\right)\right)^{4} \\
H_{24}= & 3 \varepsilon_{22}^{(1 / 3)}+3 \varepsilon_{24}^{(1 / 3)}+3 \varepsilon_{26}^{(1 / 3)}, \\
H_{25}= & \frac{3}{4 \varepsilon_{21}^{(1 / 3)}}+\frac{3 \varepsilon_{27}^{(1 / 3)}}{4}\left\|E_{0}\right\|_{F}^{(4 / 3)}(6+2 \sqrt{3})\left(k_{1}+k_{2}\right)^{(4 / 3)}+\frac{\varepsilon_{231}}{2} .
\end{aligned}
$$

Define $V_{2}(t)=V_{1}+V_{21}$. Based on the above discussion and according to (39) and (64), we have

$$
\begin{aligned}
\mathscr{L} V_{2}(t) \leq & -\left(l_{1}-H_{23}\right)\left(\widetilde{\delta}_{1}^{T} \widetilde{\delta}_{1}\right)^{2}-k_{4} e_{p}^{T} e_{p}-k_{4} e_{v}^{T} e_{v}+\frac{\varepsilon_{21}}{4}\left\|\widehat{R}_{3}\right\|^{4}\left(e_{w_{1}}^{T} e_{w_{1}}\right)^{2}+\bar{R}_{3 e}^{T} \bar{R}_{3 e} \bar{R}_{3 e}^{T} \widehat{R}_{3} w_{1 c} \\
& +\left(H_{25}+\frac{H_{24}}{4\left\|u_{1}\right\|^{(4 / 3)}}+\frac{3 \varepsilon_{25}}{4\left\|u_{1}\right\|^{2}}+\frac{1}{4 \varepsilon_{27}}\|\alpha\|_{F}^{4}\right)\left(\bar{R}_{3 e}^{T} \bar{R}_{3 e}\right)^{2}+\frac{\varepsilon_{12} T_{m}^{3}\|\alpha\|^{2}\|F(t)\|^{2} \bar{R}_{3 e}^{T} \bar{R}_{3 e} \bar{R}_{3 e}^{T} \alpha^{T} F(t)}{3 m^{3}} \\
& +\frac{\varepsilon_{232}}{2\left\|u_{1}\right\|^{4}}\left(\bar{R}_{3 e}^{T} \bar{R}_{3 e}\right)^{2}\left(e_{v}^{T} e_{v}\right)^{2}-\frac{\varepsilon_{11}}{3} \bar{R}_{3 e}^{T} \bar{R}_{3 e} \bar{R}_{3 e}^{T} E_{0} \frac{1}{\left\|u_{1}\right\|}\|F(t)\|^{2} F(t)+H_{2},
\end{aligned}
$$

where $H_{2}=H_{1}+H_{21}$. Hence, we design the virtual control law $w_{1 c}$ as

$$
\begin{aligned}
w_{1 c}= & -\left(k_{5}+H_{25}+\frac{H_{24}}{4\left\|u_{1}\right\|^{(4 / 3)}}+\frac{3 \varepsilon_{25}}{4\left\|u_{1}\right\|^{2}}+\frac{1}{4 \varepsilon_{27}}\|\alpha\|_{F}^{4}\right) \widehat{R}_{3}^{-1} \bar{R}_{3 e}-\frac{\varepsilon_{12} T_{m}^{3}\|\alpha\|^{2}\|F(t)\|^{2}}{3 m^{3}} \widehat{R}_{3}^{-1} \alpha^{T} F(t) \\
& -\frac{\varepsilon_{232}}{2\left\|u_{1}\right\|^{4}}\left(e_{v}^{T} e_{v}\right)^{2} \widehat{R}_{3}^{-1} \bar{R}_{3 e}+\frac{\varepsilon_{11}\|F(t)\|^{2}}{3\left\|u_{1}\right\|} \widehat{R}_{3}^{-1} E_{0} F(t),
\end{aligned}
$$

where $k_{5}>0$. Then, we obtain

$$
\mathscr{L} V_{2}(t) \leq-\left(L_{1}-H_{23}\right)\left(\widetilde{\delta}_{1}^{T} \widetilde{\delta}_{1}\right)^{2}-k_{4} e_{p}^{T} e_{p}-k_{4} e_{v}^{T} e_{v}-k_{5}\left(\bar{R}_{3 e}^{T} \bar{R}_{3 e}\right)^{2}+\frac{\varepsilon_{21}}{4}\left\|\widehat{R}_{3}\right\|^{4}\left(e_{w_{1}}^{T} e_{w_{1}}\right)^{2}+H_{2}
$$

From (61), the dynamic of $\bar{R}_{3 e}$ can be rewritten as 


$$
\mathrm{d} \bar{R}_{3 e}=\bar{R}_{3 e 1}(t) \mathrm{d} t+\bar{R}_{3 e 2}(t) \widetilde{\delta}_{1} \mathrm{~d} t+\bar{R}_{3 e 3}(t) \mathrm{d} \beta_{1}, \quad \text { (69) } \quad \text { where }
$$

$$
\begin{aligned}
\bar{R}_{3 e 1}(t)= & \widehat{R}_{3} e_{w_{1}}-\left(k_{5}+H_{25}+\frac{H_{24}}{4\left\|u_{1}\right\|^{(4 / 3)}}+\frac{3 \varepsilon_{25}}{4\left\|u_{1}\right\|^{2}}+\frac{1}{4 \varepsilon_{27}}\|\alpha\|_{F}^{4}\right) \bar{R}_{3 e}-\frac{\varepsilon_{12} T_{m}^{3}\|\alpha\|^{2}\|F(t)\|^{2}}{3 m^{3}} \widehat{R}_{3}^{-1} \alpha^{T} F(t) \\
& -\frac{\varepsilon_{232}}{2\left\|u_{1}\right\|^{4}}\left(e_{v}^{T} e_{v}\right)^{2} \widehat{R}_{3}^{-1} \bar{R}_{3 e}-\frac{E_{0}}{\left\|u_{1}\right\|}\left(\Delta_{101}(t)+\Delta_{102}(t) e_{v}+\Delta_{103}(t) \Delta_{104}(t)\right), \\
\bar{R}_{3 e 2}(t)= & \frac{E_{0}}{\left\|u_{1}\right\|} \Delta_{12}(t) \\
\bar{R}_{3 e 3}(t)= & -\frac{E_{0}}{\left\|u_{1}\right\|} \Delta_{13}(t) .
\end{aligned}
$$

For (37), we have

$$
\mathrm{d} e_{\psi}=\left(E_{11} w_{1}+\frac{\cos \phi}{\cos \theta} e_{r}+\frac{\cos \phi}{\cos \theta} r_{c}-\dot{\psi}_{r}\right) \mathrm{d} t
$$

where $E_{11}=(0 \sin \phi / \cos \theta)$ and $r_{c}$ is the virtual control law to be designed.

Choose Lyapunov function $V_{31}$ as

$$
V_{31}=\frac{1}{4} e_{\psi}^{4}
$$

The derivative of $V_{31}(t)$ along with (71) is derived as follows:

$$
\dot{V}_{31}(t)=e_{\psi}^{3}\left(E_{11} w_{1}+\frac{\cos \phi}{\cos \theta} e_{r}+\frac{\cos \phi}{\cos \theta} r_{c}-\dot{\psi}_{r}\right)
$$

Then, the virtual control law $r_{c}$ is designed as

$$
r_{c}=-\frac{\cos \theta}{\cos \phi}\left(E_{11} w_{1 c}-\dot{\psi}_{r}+k_{3} e_{\psi}\right) \text {. }
$$

At this point, the dynamic of $e_{\psi}$ can be written as $\mathrm{d} e_{\psi}=\left(-k_{6} e_{\psi}+E_{11} e_{w_{1}}+\frac{\cos \phi}{\cos \theta} e_{r}\right) \mathrm{d} t=\left(-k_{6} e_{\psi}+E_{1} e_{w}\right) \mathrm{d} t$

where $E_{1}=\left(\begin{array}{lll}0(\sin \phi / \cos \theta) & (\cos \phi / \cos \theta)\end{array}\right) \quad$ and $e_{w}=(p, q, r)^{T}-\left(p_{c}, q_{c}, r_{c}\right)^{T}$. Thus, based on Lemma 2 , there exists $\varepsilon_{28}>0$, such that

$$
\dot{V}_{31}(t)=e_{\psi}^{3}\left(-k_{6} e_{\psi}+E_{1} e_{w}\right)=-k_{6} e_{\psi}^{4}+e_{\psi}^{3} E_{1} e_{w} \leq-\left(k_{6}-\frac{3 \varepsilon_{28}^{(1 / 3)}}{4}\right) e_{\psi}^{4}+\frac{1}{4 \varepsilon_{28}}\left\|E_{1}\right\|^{4}\left(e_{w}^{T} e_{w}\right)^{2}
$$
yields

Define $V_{3}(t)=V_{2}(t)+V_{31}(t)$; combining (68) and (76)

$$
\begin{aligned}
\mathscr{L} V_{3}(t) \leq & -\left(l_{1}-H_{23}\right)\left(\widetilde{\delta}_{1}^{T} \widetilde{\delta}_{1}\right)^{2}-k_{4} e_{p}^{T} e_{p}-k_{4} e_{v}^{T} e_{v}-k_{5}\left(\bar{R}_{3 e}^{T} \bar{R}_{3 e}\right)+\frac{\varepsilon_{21}}{4}\left\|\widehat{R}_{3}\right\|^{4}\left\|E_{0}\right\|^{4}\left(e_{w}^{T} e_{w}\right)^{2}+H_{2} \\
& -\left(k_{6}-\frac{3 \varepsilon_{28}^{(1 / 3)}}{4}\right) e_{\psi}^{4}+\frac{1}{4 \varepsilon_{28}}\left\|E_{1}\right\|^{4}\left(e_{w}^{T} e_{w}\right)^{2} .
\end{aligned}
$$

In what follows, consider the dynamic of angular velocity:

$$
J \mathrm{~d} w=\left(-w^{\times} J w+A\left(T_{m}\right) \rho+B\left(T_{m}\right)+\delta_{2}\right) \mathrm{d} t+G_{2} \mathrm{~d} \beta_{2}
$$


Defining $\rho=A\left(T_{m}\right)^{-1}\left(u_{2}-B\left(T_{m}\right)+w^{\times} J w-\widehat{\delta}_{2}\right)$, the where $\mathrm{d} w_{c}$ is obtained in Appendix. Then, according to (79), error dynamic of $e_{w}$ is

$$
J \mathrm{~d} e_{w}=\left(u_{2}+\widetilde{\delta}_{2}\right) \mathrm{d} t+G_{2} \mathrm{~d} \beta_{2}-\mathrm{d} w_{c}
$$

$$
J \mathrm{~d} e_{w}=\left(u_{2}+\widetilde{\delta}_{2}\right) \mathrm{d} t+G_{2} \mathrm{~d} \beta_{2}-W_{c 1}(t) \mathrm{d} t-W_{c 2}(t) \widetilde{\delta}_{1} \mathrm{~d} t-W_{c 20}\left(t, \widetilde{\delta}_{1}\right) \mathrm{d} t-W_{c 3}(t) \mathrm{d} \beta_{1}
$$

which implies that

$$
\mathrm{d} e_{w}=\left(J^{-1} u_{2}-J^{-1} W_{c 1}(t)-J^{-1} W_{c 2}(t) \widetilde{\delta}_{1}-J^{-1} W_{c 20}\left(t, \widetilde{\delta}_{1}\right)+J^{-1} \widetilde{\delta}_{2}\right) \mathrm{d} t-J^{-1} W_{c 3}(t) \mathrm{d} \beta_{1}+J^{-1} G_{2} \mathrm{~d} \beta_{2}
$$

Choose Lyapunov function as follows:

$$
V_{41}(t)=\frac{1}{4}\left(e_{w}^{T} e_{w}\right)^{2}
$$

The infinitesimal generator of $V_{41}$ along with (81) is obtained as follows:

$$
\begin{aligned}
\mathscr{L} V_{41}(t)= & e_{w}^{T} e_{w} e_{w}^{T} J^{-1} u_{2}-e_{w}^{T} e_{w} e_{w}^{T} J^{-1} W_{c 1}(t)-e_{w}^{T} e_{w} e_{w}^{T} J^{-1} W_{c 2}(t) \widetilde{\delta}_{1}-e_{w}^{T} e_{w} e_{w}^{T} J^{-1} W_{c 20}\left(t, \widetilde{\delta}_{1}\right) \\
& +e_{w}^{T} e_{w} e_{w}^{T} J^{-1} \widetilde{\delta}_{2}+\frac{1}{2} \operatorname{Tr}\left(W_{c 3}^{T}(t) J^{-T}\left(2 e_{w} e_{w}^{T}+e_{w}^{T} e_{w} I\right) J^{-1} W_{c 3}(t)\right) \\
& +\frac{1}{2} \operatorname{Tr}\left(G_{2}^{T} J^{-T}\left(2 e_{w} e_{w}^{T}+e_{w}^{T} e_{w} I\right) J^{-1} G_{2}\right) .
\end{aligned}
$$

From Lemma 2, there exist parameters $\varepsilon_{31}>0, \varepsilon_{32}>0$, $\varepsilon_{33}>0, \varepsilon_{34}>0$, and $\varepsilon_{35}>0$ such that

$$
\begin{gathered}
-e_{w}^{T} e_{w} e_{w}^{T} J^{-1} W_{c 2}(t) \widetilde{\delta}_{1} \leq \frac{3}{4 \varepsilon_{31}^{(1 / 3)}}\left\|J^{-1}\right\|_{F}^{(4 / 3)}\left\|W_{c 2}(t)\right\|_{F}^{(4 / 3)}\left(e_{w}^{T} e_{w}\right)^{2}+\frac{\varepsilon_{31}}{4}\left(\widetilde{\delta}_{1}^{T} \widetilde{\delta}_{1}\right)^{2}, \\
e_{w}^{T} e_{w} e_{w}^{T} J^{-1} \widetilde{\delta}_{2} \leq \frac{3}{4 \varepsilon_{32}^{(1 / 3)}}\left\|J^{-1}\right\|_{F}^{(4 / 3)}\left(e_{w}^{T} e_{w}\right)^{2}+\frac{\varepsilon_{32}}{4}\left(\widetilde{\delta}_{2}^{T} \widetilde{\delta}_{2}\right)^{2}, \\
\frac{1}{2} \operatorname{Tr}\left(W_{c 3}^{T}(t) J^{-T}\left(2 e_{w} e_{w}^{T}+e_{w}^{T} e_{w} I\right) J^{-1} W_{c 3}(t)\right) \leq \frac{3 \varepsilon_{33}}{4}\left\|J^{-1}\right\|_{F}^{4}\left\|W_{c 3}(t)\right\|^{4}\left(e_{w}^{T} e_{w}\right)^{2}+\frac{3}{4 \varepsilon_{33}}, \\
\frac{1}{2} \operatorname{Tr}\left(G_{2}^{T} J^{-T}\left(2 e_{w} e_{w}^{T}+e_{w}^{T} e_{w} I\right) J^{-1} G_{2}\right) \leq \frac{3 \varepsilon_{34}}{4}\left\|J^{-1}\right\|_{F}^{4}\left\|G_{2}\right\|^{4}\left(e_{w}^{T} e_{w}\right)^{2}+\frac{3}{4 \varepsilon_{34}}, \\
-e_{w}^{T} e_{w} e_{w}^{T} J^{-1} W_{c 20}\left(t, \widetilde{\delta}_{1}\right) \leq \frac{3}{4 \varepsilon_{35}^{(1 / 3)}} \Pi\left(e_{w}^{T} e_{w}\right)^{2}+\frac{\varepsilon_{35}}{2}\left(\widetilde{\delta}_{1}^{T} \widetilde{\delta}_{1}\right)^{2},
\end{gathered}
$$

where $\quad \Pi=\left\|J^{-1}\right\|\left\|W_{c 21}(t)\right\|^{(4 / 3)}\left\|\Delta_{502}(t)\right\|^{(4 / 3)}\left\|\alpha_{31}\right\|^{(4 / 3)} \| F$ $(t)\left\|^{(4 / 3)}+\right\| J^{-1}\|\quad\| W_{c 21}(t)\left\|^{(4 / 3)}\right\| \Delta_{512}(t)\left\|^{(4 / 3)}\right\| \alpha_{32}\left\|^{(4 / 3)}\right\| F$

$(t) \|^{(4 / 3)}$. Hence, based on (83), we get 


$$
\begin{aligned}
\mathscr{L} V_{41}(t) \leq & e_{w}^{T} e_{w} e_{w}^{T} J^{-1} u_{2}-e_{w}^{T} e_{w} e_{w}^{T} J^{-1} W_{c 1}(t)+\frac{3}{4 \varepsilon_{31}^{(1 / 3)}}\left\|J^{-1}\right\|_{F}^{(4 / 3)}\left\|W_{c 2}(t)\right\|_{F}^{(4 / 3)}\left(e_{w}^{T} e_{w}\right)^{2}+\frac{\varepsilon_{31}}{4}\left(\widetilde{\delta}_{1}^{T} \widetilde{\delta}_{1}\right)^{2} \\
& +\frac{3}{4 \varepsilon_{32}^{(1 / 3)}}\left\|J^{-1}\right\|_{F}^{(4 / 3)}\left(e_{w}^{T} e_{w}\right)^{2}+\frac{\varepsilon_{32}}{4}\left(\widetilde{\delta}_{2}^{T} \widetilde{\delta}_{2}\right)^{2}+\frac{3 \varepsilon_{33}}{4}\left\|J^{-1}\right\|_{F}^{4}\left\|W_{c 3}(t)\right\|^{4}\left(e_{w}^{T} e_{w}\right)^{2}+\frac{3}{4 \varepsilon_{33}} \\
& +\frac{3 \varepsilon_{34}}{4}\left\|J^{-1}\right\|_{F}^{4}\left\|G_{2}\right\|^{4}\left(e_{w}^{T} e_{w}\right)^{2}+\frac{3}{4 \varepsilon_{34}}+\frac{3}{4 \varepsilon_{35}^{(1 / 3)}} \Pi\left(e_{w}^{T} e_{w}\right)^{2}+\frac{\varepsilon_{35}}{4}\left(\widetilde{\delta}_{1}^{T} \widetilde{\delta}_{1}\right)^{2}
\end{aligned}
$$

Design the controller $u_{2}$ as

$$
\begin{aligned}
u_{2}= & -\left(k_{7}+\frac{3}{4 \varepsilon_{31}^{(1 / 3)}}\left\|J^{-1}\right\|_{F}^{(4 / 3)}\left\|W_{c 2}(t)\right\|_{F}^{(4 / 3)}+\frac{3}{4 \varepsilon_{32}^{(1 / 3)}}\left\|J^{-1}\right\|_{F}^{(4 / 3)}+\frac{3 \varepsilon_{33}}{4}\left\|J^{-1}\right\|_{F}^{4}\left\|W_{c 3}(t)\right\|^{4}\right. \\
& \left.+\frac{3 \varepsilon_{34}}{4}\left\|J^{-1}\right\|_{F}^{4}\left\|G_{2}\right\|^{4}+\frac{3}{4 \varepsilon_{35}^{(1 / 3)}} \Pi\right) e_{w}+W_{c 1}(t)+u_{3},
\end{aligned}
$$

where $u_{3}$ is designed in the following steps.

$\mathscr{L} V_{41}(t)$ can be written by

$$
\mathscr{L} V_{41}(t) \leq-k_{7}\left(e_{w}^{T} e_{w}\right)^{2}+\left(\frac{\varepsilon_{31}}{4}+\frac{\varepsilon_{35}}{4}\right)\left(\widetilde{\delta}_{1}^{T} \widetilde{\delta}_{1}\right)^{2}+\frac{\varepsilon_{32}}{4}\left(\widetilde{\delta}_{2}^{T} \widetilde{\delta}_{2}\right)^{2}+e_{w}^{T} e_{w} e_{w}^{T} J^{-1} u_{2}+\frac{3}{4 \varepsilon_{33}}+\frac{3}{4 \varepsilon_{34}}
$$

Define Lyapunov function as follows:

$$
V(t)=V_{02}(t)+V_{3}(t)+V_{41}
$$

Combining (44), (77), and (87), the infinitesimal generator of (88) is represented as

$$
\begin{aligned}
\mathscr{L} V(t) \leq & -\left(l_{1}-H_{23}-\frac{\varepsilon_{31}}{4}-\frac{\varepsilon_{35}}{4}\right)\left(\widetilde{\delta}_{1}^{T} \widetilde{\delta}_{1}\right)^{2}-\left(l_{2}-\frac{\varepsilon_{32}}{4}\right)\left(\widetilde{\delta}_{2}^{T} \widetilde{\delta}_{2}\right)^{2}-k_{4} e_{p}^{T} e_{p}-k_{4} e_{v}^{T} e_{v} \\
& -k_{5}\left(\bar{R}_{3 e}^{T} \bar{R}_{3 e}\right)^{2}-\left(k_{6}-\frac{3 \varepsilon_{28}^{(1 / 3)}}{4}\right) e_{\psi}^{4}-k_{7}\left(e_{w}^{T} e_{w}\right)^{2}+e_{w}^{T} e_{w} e_{w}^{T} J^{-1} u_{3} \\
& +\frac{\varepsilon_{21}}{4}\left\|\widehat{R}_{3}\right\|_{F}^{4}\left\|E_{0}\right\|^{4}\left(e_{w_{1}}^{T} e_{w_{1}}\right)^{2}+\frac{1}{4 \varepsilon_{28}}\left\|E_{1}\right\|^{4}\left(e_{w}^{T} e_{w}\right)^{2}+H_{3}, \\
u_{3} & =-\left(\frac{\varepsilon_{21}}{4}\left\|\widehat{R}_{3}\right\|_{F}^{4}\left\|E_{0}\right\|^{4}+\frac{1}{4 \varepsilon_{28}}\left\|E_{1}\right\|^{4}\right) J e_{w} .
\end{aligned}
$$

where

$$
H_{3}=H_{2}+\frac{3}{4 \varepsilon_{33}}+\frac{3}{4 \varepsilon_{34}}+H_{02}
$$

Recalling that $e_{w_{1}}^{T} e_{w_{1}} \leq e_{w}^{T} e_{w}, u_{3}$ is designed as

$$
\begin{aligned}
\mathscr{L} V(t) \leq & -\left(l_{1}-H_{23}-\frac{\varepsilon_{31}}{4}-\frac{\varepsilon_{35}}{4}\right)\left(\widetilde{\delta}_{1}^{T} \widetilde{\delta}_{1}\right)^{2}-\left(l_{2}-\frac{\varepsilon_{32}}{4}\right)\left(\widetilde{\delta}_{2}^{T} \widetilde{\delta}_{2}\right)^{2}-k_{4} e_{p}^{T} e_{p} \\
& -k_{4} e_{v}^{T} e_{v}-k_{5}\left(\bar{R}_{3 e}^{T} \bar{R}_{3 e}\right)^{2}-\left(k_{6}-\frac{3 \varepsilon_{28}^{(1 / 3)}}{4}\right) e_{\psi}^{4}-k_{7}\left(e_{w}^{T} e_{w}\right)^{2}+H_{3} .
\end{aligned}
$$


Based on (86) and (91), (81) can be written as

$$
\begin{aligned}
J \mathrm{~d} e_{w}= & -\left(k_{7}+\frac{3}{4 \varepsilon_{31}^{(1 / 3)}}\left\|J^{-1}\right\|_{F}^{(4 / 3)}\left\|W_{c 2}(t)\right\|_{F}^{(4 / 3)}+\frac{3}{4 \varepsilon_{32}^{(1 / 3)}}\left\|J^{-1}\right\|_{F}^{(4 / 3)}+\frac{3 \varepsilon_{33}}{4}\left\|J^{-1}\right\|_{F}^{4}\left\|W_{c 3}(t)\right\|^{4}+\frac{3 \varepsilon_{34}}{4}\left\|J^{-1}\right\|_{F}^{4}\left\|G_{2}\right\|^{4}\right. \\
& \left.+\frac{3}{4 \varepsilon_{35}^{(1 / 3)}} \Pi\right) e_{w} \mathrm{~d} t-\left(\frac{\varepsilon_{21}}{4}\left\|\widehat{R}_{3}\right\|_{F}^{4}\left\|E_{0}\right\|^{4}+\frac{1}{4 \varepsilon_{28}}\left\|E_{1}\right\|^{4}\right) J e_{w} \mathrm{~d} t-W_{c 20}\left(t, \widetilde{\delta}_{1}\right) \mathrm{d} t+\widetilde{\delta}_{2} \mathrm{~d} t \\
& -W_{c 3}(t) \mathrm{d} \beta_{1}+G_{2} \mathrm{~d} \beta_{2} .
\end{aligned}
$$

By using (40), (41), (22), (27), (68), (75), and (93), we get

$$
\begin{aligned}
\mathrm{d} \widetilde{\delta}_{1}= & -\left(L_{1}+\left(\frac{3 \varepsilon_{01}^{(4 / 3)}}{4}+\frac{3 \varepsilon_{02}^{2}}{4}\left\|L_{1} G_{1}\right\|_{F}^{4}\right)\right) \widetilde{\delta}_{1} \mathrm{~d} t-L_{1} G_{1} \mathrm{~d} \beta_{1}-\dot{\delta}_{1} \mathrm{~d} t-\frac{\varepsilon_{11}}{3}\|F(t)\|^{2} F(t)^{T} \mathrm{~d} t, \\
\mathrm{~d} \widetilde{\delta}_{2}= & -\left(l_{2}+\frac{3 \varepsilon_{03}^{(4 / 3)}}{4}+\frac{3 \varepsilon_{04}^{2}}{4}\left\|L_{2} G_{2}\right\|_{F}^{4}\right) \widetilde{\delta}_{2} \mathrm{~d} t-\dot{\delta}_{2} \mathrm{~d} t-L_{2} G_{2} \mathrm{~d} \beta_{2}, \\
\mathrm{~d} e_{p}= & e_{v} \mathrm{~d} t, \\
\mathrm{~d} e_{v}= & \left(-k_{1} \tanh \left(e_{p}+e_{v}\right)-k_{2} \tanh \left(e_{v}\right)+\frac{T_{m}}{m} \alpha \bar{R}_{3 e}+\widetilde{\delta}_{1}\right) \mathrm{d} t+G_{1} \mathrm{~d} \beta_{1}, \\
\mathrm{~d} e_{\psi}= & \left(-k_{6} e_{\psi}+E_{1} e_{w}\right) \mathrm{d} t, \\
\mathrm{~d} \bar{R}_{3 e}= & \bar{R}_{3 e 1}(t) \mathrm{d} t+\bar{R}_{3 e 2}(t) \widetilde{\delta}_{1}+\bar{R}_{3 e 3}(t) \mathrm{d} \beta_{1}, \\
J \mathrm{~d} e_{w}= & -\left(k_{7}+\frac{3}{4 \varepsilon_{31}^{(1 / 3)}}\left\|J^{-1}\right\|_{F}^{(4 / 3)}\left\|W_{c 2}(t)\right\|_{F}^{(4 / 3)}+\frac{3}{4 \varepsilon_{32}^{(1 / 3)}}\left\|J^{-1}\right\|_{F}^{(4 / 3)}+\frac{3 \varepsilon_{33}}{4}\left\|J^{-1}\right\|_{F}^{4}\left\|W_{c 3}(t)\right\|^{4}+\frac{3 \varepsilon_{34}}{4}\left\|J^{-1}\right\|_{F}^{4}\left\|G_{2}\right\|^{4}\right. \\
& \left.+\frac{3}{4 \varepsilon_{35}^{(1 / 3)}} \Pi\right) e_{w} \mathrm{~d} t-\left(\frac{\varepsilon_{21}}{4}\left\|\widehat{R}_{3}\right\|_{F}^{4}\left\|E_{0}\right\|^{4}+\frac{1}{4 \varepsilon_{28}}\left\|E_{1}\right\|^{4}\right) J e_{w} \mathrm{~d} t-W_{c 20}\left(t, \widetilde{\delta}_{1}\right) \mathrm{d} t+\widetilde{\delta}_{2} \mathrm{~d} t \\
& -W_{c 3}(t) \mathrm{d} \beta_{1}+G_{2} \mathrm{~d} \beta_{2} .
\end{aligned}
$$

Remark 2. In this paper, the dynamics of helicopter are modeled as the stochastic systems due to the disturbances including the random disturbances. Many control variables of the helicopter stochastic systems are discontinuous. Hence, some advanced control methods cannot be used to design the flight controller, directly, such as the dynamic surface control, fuzzy control, and other control methods. In order to construct the antidisturbance flight controller for the helicopter stochastic systems, the disturbance observer method and backstepping control scheme are applied in our paper.

Remark 3. The control method proposed in this paper shows some weaknesses, such as the complicated controller structure and tedious mathematical derivation while the helicopter stochastic system models are first discussed in this paper and the nonlinear control method is feasible in theory. In the future, we will construct the more advanced antidisturbance flight controller with the simple structure based on the proposed control scheme of this paper.

Remark 4. The structure block diagram of the antidisturbance control scheme is shown in Figure 1. The noises from inside and outside of the helicopter are considered to construct the helicopter models and divided as to different parts: the random disturbances and other times-varying disturbances. Then, the stochastic control theory is applied to analyze the stability of the closed-loop systems. And the stochastic antidisturbance flight controller is constructed. 


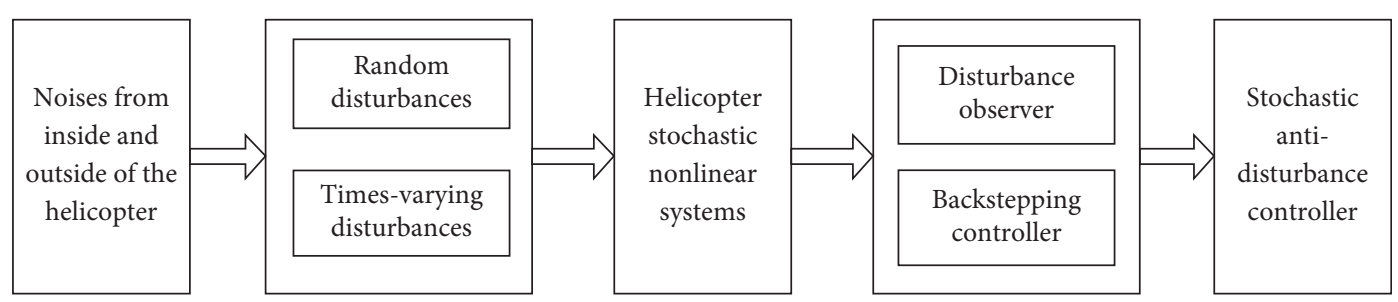

FIgURE 1: The structure block diagram of the antidisturbance control scheme.

\section{Stability Analysis}

In this section, the asymptotic boundedness in mean square of the composite closed-loop system (94) is guaranteed under the controllers $u_{1}$ and $u_{2}$.

Theorem 1. Considering helicopter system (1) with disturbances, design the disturbance observers as (10) and (41) and the controllers as (20) and (86). The composite closed-loop system (94) is asymptotically bounded in mean square. If there exist parameters $k_{1}>0, k_{2}>0, k_{3}>0, k_{4}>0, k_{5}>0, k_{6}>0$, $k_{7}>0, k_{8}>0, k_{9}>0, l_{1}>0, l_{2}>0, \varepsilon_{01}>0, \varepsilon_{02}>0, \varepsilon_{03}>0$, $\varepsilon_{04}>0, \varepsilon_{11}>0, \varepsilon_{12}>0, \varepsilon_{21}>0, \varepsilon_{22}>0, \varepsilon_{23}>0, \varepsilon_{231}>0, \varepsilon_{232}>0$, $\varepsilon_{24}>0, \varepsilon_{25}>0, \varepsilon_{26}>0, \varepsilon_{27}>0, \varepsilon_{28}>0, \varepsilon_{31}>0, \varepsilon_{32}>0, \varepsilon_{33}>0$, $\varepsilon_{34}>0$, and $\varepsilon_{35}>0$, such that $l_{1}-H_{23}-\left(\varepsilon_{31} / 4\right)-\left(\varepsilon_{35} / 4\right)>0$, $l_{2}-\left(\varepsilon_{32} / 4\right)>0, k_{4}-H_{22}>0, k_{6}-\left(3 \varepsilon_{28}^{(1 / 3)} / 4\right)>0$ hold.

Proof. Consider the Lyapunov function $V(t)$ as follows:

$$
\begin{aligned}
V(t)= & V_{02}(t)+V_{3}(t)+V_{41}(t) \\
= & \frac{1}{4}\left(\widetilde{\delta}_{1}^{T} \widetilde{\delta}_{1}\right)^{2}+\frac{1}{4}\left(\widetilde{\delta}_{2}^{T} \widetilde{\delta}_{2}\right)^{2}+k_{1} \int_{0}^{e_{p}+e_{v}} \tanh (\mu) \mathrm{d} \mu+k_{2} \int_{0}^{e_{v}} \tanh (\mu) \mathrm{d} \mu \\
& +\frac{1}{2} e_{v}^{T} e_{v}+\frac{1}{4}\left(\bar{R}_{3 e}^{T} \bar{R}_{3 e}\right)^{2}+\frac{1}{4} e_{\psi}^{4}+\frac{1}{4}\left(e_{w}^{T} e_{w}\right)^{2} .
\end{aligned}
$$

Since $h(x)=(\tanh (x) / x)$ is a continuous function, for any $\bar{x}>0$ and $0 \leq x \leq \bar{x}$, there exists a positive number $\beta_{1}=\beta(\bar{x})$, such that $\beta_{1}\|x\| \leq\|\tanh (x)\| \leq\|x\|$. Hence, we have

$$
k_{1} \int_{0}^{e_{p}+e_{v}} \tanh (\mu) \mathrm{d} \mu+k_{2} \int_{0}^{e_{v}} \tanh (\mu) \mathrm{d} \mu \geq k_{8} e_{p}^{T} e_{p}+k_{8} e_{v}^{T} e_{v},
$$

where $k_{8}=\max \left\{\operatorname{eig}\left\{\left(\begin{array}{cc}(1 / 2) k_{1} \beta_{1} & (1 / 2) k_{1} \beta_{1} \\ (1 / 2) k_{1} \beta_{1} & (1 / 2) k_{1} \beta_{1}+(1 / 2) k_{2} \beta_{1}\end{array}\right)\right\}\right\}>$
0. Moreover,

$$
k_{1} \int_{0}^{e_{p}+e_{v}} \tanh (\mu) \mathrm{d} \mu+k_{2} \int_{0}^{e_{v}} \tanh (\mu) \mathrm{d} \mu \leq k_{9} e_{p}^{T} e_{p}+k_{9} e_{v}^{T} e_{v},
$$

where $k_{9}=\min \left\{\operatorname{eig}\left\{\left(\begin{array}{cc}(1 / 2) k_{1} & (1 / 2) k_{1} \\ (1 / 2) k_{1} & (1 / 2) k_{1}+(1 / 2) k_{2}\end{array}\right)\right\}\right\}>0$.

Then, we confirm that

$$
\begin{aligned}
& \frac{1}{4}\left(\widetilde{\delta}_{1}^{T} \widetilde{\delta}_{1}\right)^{2}+\frac{1}{4}\left(\widetilde{\delta}_{2}^{T} \widetilde{\delta}_{2}\right)^{2}+k_{8} e_{p}^{T} e_{p}+\left(k_{8}+\frac{1}{2}\right) e_{v}^{T} e_{v}+\frac{1}{4}\left(\bar{R}_{3 e}^{T} \bar{R}_{3 e}\right)^{2}+\frac{1}{4} e_{\psi}^{4}+\frac{1}{4}\left(e_{w}^{T} e_{w}\right)^{2} \\
& \quad \leq V(t) \leq \frac{1}{4}\left(\widetilde{\delta}_{1}^{T} \widetilde{\delta}_{1}\right)^{2}+\frac{1}{4}\left(\widetilde{\delta}_{2}^{T} \widetilde{\delta}_{2}\right)^{2}+k_{9} e_{p}^{T} e_{p}+\left(k_{9}+\frac{1}{2}\right) e_{v}^{T} e_{v}+\frac{1}{4}\left(\bar{R}_{3 e}^{T} \bar{R}_{3 e}\right)^{2}+\frac{1}{4} e_{\psi}^{4}+\frac{1}{4}\left(e_{w}^{T} e_{w}\right)^{2},
\end{aligned}
$$

which satisfies (8) in Lemma 1 with $p=2$.

From the discussion in Sections 3 and 4, we have

$$
\begin{aligned}
\mathscr{L} V(t) \leq & -\left(l_{1}-H_{23}-\frac{\varepsilon_{31}}{4}-\frac{\varepsilon_{35}}{4}\right)\left(\widetilde{\delta}_{1}^{T} \widetilde{\delta}_{1}\right)^{2}-\left(l_{2}-\frac{\varepsilon_{32}}{4}\right)\left(\widetilde{\delta}_{2}^{T} \widetilde{\delta}_{2}\right)^{2}-k_{4} e_{p}^{T} e_{p} \\
& -k_{4} e_{v}^{T} e_{v}-k_{5}\left(\bar{R}_{3 e}^{T} \bar{R}_{3 e}\right)^{2}-\left(k_{6}-\frac{3 \varepsilon_{28}^{(1 / 3)}}{4}\right) e_{\psi}^{4}-k_{7}\left(e_{w}^{T} e_{w}\right)^{2}+H_{3} \leq-\lambda V(t)+H_{3},
\end{aligned}
$$


where

$$
\lambda=\min \left\{4\left(l_{1}-H_{23}-\frac{\varepsilon_{31}}{4}-\frac{\varepsilon_{35}}{4}\right), 4\left(l_{2}-\frac{\varepsilon_{32}}{4}\right), \frac{k_{4}}{k_{9}}, \frac{k_{4}}{k_{9}+(1 / 2)}, 4 k_{5}, 4\left(k_{6}-\frac{3 \varepsilon_{28}^{(1 / 3)}}{4}\right), 4 k_{7}\right\}
$$

which implies that $V(t)$ satisfies (9) in Lemma 1 with $p=2$.

Consequently, based on the discussion above, the composite closed-loop system (94) is asymptotically bounded in mean square.

\section{Conclusion}

This paper studies the tracking control issue for nonlinear helicopter systems under stochastic disturbances and timevarying disturbances. These disturbances are attenuated and rejected via the nonlinear disturbance observer control method and stochastic control theory, and the backstepping control approach is adopted for nonlinear helicopter systems. The problems of "dimension explosion" are analyzed for complicated nonlinear control systems.

Since the helicopter systems are modeled as the stochastic systems, many advanced nonlinear control methods can be directly used. The phenomenon of "dimension explosion" is inevitable in nonlinear controller constructing process. In the future, we will combine some advanced nonlinear control methods and stochastic control theory and propose the more advanced antidisturbance flight control scheme with simple structure.

\section{Appendix}

From (67), $\mathrm{d} w_{1 c}$ can be written by

$$
\begin{aligned}
\mathrm{d} w_{1 c}= & -\mathrm{d}\left(\left(k_{5}+H_{25}+\frac{H_{24}}{4\left\|u_{1}\right\|^{(4 / 3)}}+\frac{3 \varepsilon_{25}}{4\left\|u_{1}\right\|^{2}}+\frac{1}{4 \varepsilon_{27}}\|\alpha\|_{F}^{4}\right) \widehat{R}_{3}^{-1} \bar{R}_{3 e}\right) \\
& -\mathrm{d}\left(\frac{\varepsilon_{12}\left\|u_{1}\right\|^{3}\|\alpha\|^{2}\|F(t)\|^{2}}{3} \widehat{R}_{3}^{-1} \alpha^{T} F(t)\right) \\
& -\mathrm{d}\left(\frac{\varepsilon_{232}}{2\left\|u_{1}\right\|^{4}}\left(e_{v}^{T} e_{v}\right)^{2} \widehat{R}_{3}^{-1} \bar{R}_{3 e}\right)+d\left(\frac{\varepsilon_{11}\|F(t)\|^{2}}{3\left\|u_{1}\right\|} \widehat{R}_{3}^{-1} E_{0} F(t)\right) .
\end{aligned}
$$

\section{A. The Calculation of $\mathrm{d}\left(\left(k_{5}+H_{25}+\left(H_{24} / 4\left\|u_{1}\right\|\right.\right.\right.$}

$\left.\left.(4 / 3))+\left(3 \boldsymbol{\varepsilon}_{25} / 4\left\|u_{1}\right\|^{2}\right)+\left(1 / 4 \boldsymbol{\varepsilon}_{27}\right)\left\|u_{1}\right\|_{F}^{4}\right) \widehat{R}_{3}^{-1} \bar{R}_{3 e}\right)$

Consider

$$
\begin{gathered}
\mathrm{d}\left(\left(k_{5}+H_{25}+\frac{H_{24}}{4\left\|u_{1}\right\|^{(4 / 3)}}+\frac{3 \varepsilon_{25}}{4\left\|u_{1}\right\|^{2}}+\frac{1}{4 \varepsilon_{27}}\|\alpha\|_{F}^{4}\right) \widehat{R}_{3}^{-1} \bar{R}_{3 e}\right) \\
\quad=\mathrm{d}\left(k_{5}+H_{25}+\frac{H_{24}}{4\left\|u_{1}\right\|^{(4 / 3)}}+\frac{3 \varepsilon_{25}}{4\left\|u_{1}\right\|^{2}}+\frac{1}{4 \varepsilon_{27}}\|\alpha\|_{F}^{4}\right) \widehat{R}_{3}^{-1} \bar{R}_{3 e} \\
\left(k_{5}+H_{25}+\frac{H_{24}}{4\left\|u_{1}\right\|^{(4 / 3)}}+\frac{3 \varepsilon_{25}}{4\left\|u_{1}\right\|^{2}}+\frac{1}{4 \varepsilon_{27}}\|\alpha\|_{F}^{4}\right) \mathrm{d} \widehat{R}_{3}^{-1} \bar{R}_{3 e} \\
\left(k_{5}+H_{25}+\frac{H_{24}}{4\left\|u_{1}\right\|^{(4 / 3)}}+\frac{3 \varepsilon_{25}}{4\left\|u_{1}\right\|^{2}}+\frac{1}{4 \varepsilon_{27}}\|\alpha\|_{F}^{4}\right) \widehat{R}_{3}^{-1} \mathrm{~d} \bar{R}_{3 e} .
\end{gathered}
$$

For $\mathrm{d} u_{1}$, we deduce 


$$
\mathrm{d}\left\|u_{1}\right\|=-\frac{\mathrm{d} u_{1}^{T} \cdot u_{1}+u_{1}^{T} \mathrm{~d} u_{1}}{2 \sqrt{u_{1}^{T} u_{1}}}=-\frac{u_{1}^{T}}{\left\|u_{1}\right\|}\left(\Delta_{01}(t) \mathrm{d} t+\Delta_{02}(t) \widetilde{\delta}_{1} \mathrm{~d} t+\Delta_{03}(t) \mathrm{d} \beta_{1}\right)
$$

which means that

$$
\begin{gathered}
\mathrm{d} \frac{H_{24}}{4\left\|u_{1}\right\|^{(4 / 3)}}=-\frac{H_{24}}{3\left\|u_{1}\right\|^{(7 / 3)}} \mathrm{d}\left\|u_{1}\right\|=\frac{H_{24} u_{1}^{T}}{3\left\|u_{1}\right\|^{(10 / 3)}}\left(\Delta_{01}(t) \mathrm{d} t+\Delta_{02}(t) \widetilde{\delta}_{1} \mathrm{~d} t+\Delta_{03}(t) \mathrm{d} \beta_{1}\right), \\
\mathrm{d} \frac{3 \varepsilon_{25}}{4\left\|u_{1}\right\|^{2}}=-\frac{3 \varepsilon_{25}}{2\left\|u_{1}\right\|^{3}} \mathrm{~d}\left\|u_{1}\right\|=\frac{3 \varepsilon_{25} u_{1}^{T}}{2\left\|u_{1}\right\|^{4}}\left(\Delta_{01}(t) \mathrm{d} t+\Delta_{02}(t) \widetilde{\delta}_{1} \mathrm{~d} t+\Delta_{03}(t) \mathrm{d} \beta_{1}\right) .
\end{gathered}
$$

For $\|\alpha\|_{F}$, one has

$$
\alpha^{T} \alpha=\left(\begin{array}{ccc}
1 & 0 & \frac{-R_{13}-R_{13 c}}{R_{33}+R_{33 c}} \\
0 & 1 & \frac{-R_{23}-R_{23 c}}{R_{33}+R_{33 c}}
\end{array}\right)\left(\begin{array}{cc}
1 & 0 \\
0 & 1 \\
\frac{-R_{13}-R_{13 c}}{R_{33}+R_{33 c}} & \frac{-R_{23}-R_{23 c}}{R_{33}+R_{33 c}}
\end{array}\right)=\left(\begin{array}{cc}
1+\left(\frac{R_{13}+R_{13 c}}{R_{33}+R_{33 c}}\right)^{2} & \frac{\left(R_{13}+R_{13 c}\right)\left(R_{23}+R_{23 c}\right)}{\left(R_{33}+R_{33 c}\right)^{2}} \\
\frac{\left(R_{13}+R_{13 c}\right)\left(R_{23}+R_{23 c}\right)}{\left(R_{33}+R_{33 c}\right)^{2}} & 1+\left(\frac{R_{23}+R_{23 c}}{R_{33}+R_{33 c}}\right)^{2}
\end{array}\right) .
$$

Hence,

$$
\begin{aligned}
\|\alpha\|_{F}= & \left(\operatorname{Tr}\left(\alpha \alpha^{T}\right)\right)^{(1 / 2)}=\left(\operatorname{Tr}\left(\alpha^{T} \alpha\right)\right)^{(1 / 2)}=2+\frac{\left(R_{13}+R_{13 c}\right)^{2}+\left(R_{23}+R_{23 c}\right)^{2}}{\left(R_{33}+R_{33 c}\right)^{2}}, \\
d\|\alpha\|_{F}= & \Delta_{21}(t) \mathrm{d} R_{3}+\Delta_{21}(t) \frac{1}{\sqrt{u_{1}^{T} u_{1}}}\left(I_{3}-\frac{u_{1} u_{1}^{T}}{u_{1}^{T} u_{1}}\right) \Delta_{01}(t) \mathrm{d} t+\Delta_{21}(t) \frac{1}{\sqrt{u_{1}^{T} u_{1}}}\left(I_{3}-\frac{u_{1} u_{1}^{T}}{u_{1}^{T} u_{1}}\right) \Delta_{02}(t) \widetilde{\delta}_{1} \mathrm{~d} t \\
& +\Delta_{21}(t) \frac{1}{\sqrt{u_{1}^{T} u_{1}}}\left(I_{3}-\frac{u_{1} u_{1}^{T}}{u_{1}^{T} u_{1}}\right) \Delta_{03}(t) \mathrm{d} \beta_{1},
\end{aligned}
$$

where

$$
\Delta_{21}(t)=\left(\frac{2\left(R_{13}+R_{13 c}\right)}{\left(R_{33}+R_{33 c}\right)^{2}} \frac{2\left(R_{23}+R_{23 c}\right)}{\left(R_{33}+R_{33 c}\right)^{2}} \frac{2\left(R_{13}+R_{13 c}\right)^{2}+\left(R_{23}+R_{23 c}\right)^{2}}{\left(R_{33}+R_{33 c}\right)^{2}}\right) .
$$

From the above discussion,

$$
\mathrm{d}\left(k_{5}+H_{25}+\frac{H_{24}}{4\left\|u_{1}\right\|^{(4 / 3)}}+\frac{3 \varepsilon_{25}}{4\left\|u_{1}\right\|^{2}}+\frac{1}{4 \varepsilon_{27}}\|\alpha\|_{F}^{4}\right)=\Delta_{31}(t) \mathrm{d} t+\Delta_{32}(t) \widetilde{\delta}_{1} \mathrm{~d} t+\Delta_{33}(t) \mathrm{d} \beta_{1},
$$


where

$$
\begin{aligned}
& \Delta_{31}(t)=\frac{H_{24} u_{1}^{T}}{3\left\|u_{1}\right\|^{(10 / 3)}} \Delta_{01}(t)+\frac{3 \varepsilon_{25} u_{1}^{T}}{2\left\|u_{1}\right\|^{4}} \Delta_{01}(t)+\frac{1}{\varepsilon_{27}}\|\alpha\|_{F}^{3} \Delta_{22}(t), \\
& \Delta_{32}(t)=\frac{H_{24} u_{1}^{T}}{3\left\|u_{1}\right\|^{(10 / 3)}} \Delta_{02}(t)+\frac{3 \varepsilon_{25} u_{1}^{T}}{2\left\|u_{1}\right\|^{4}} \Delta_{02}(t)+\frac{1}{\varepsilon_{27}}\|\alpha\|_{F}^{3} \Delta_{23}(t), \\
& \Delta_{33}(t)=\frac{H_{24} u_{1}^{T}}{3\left\|u_{1}\right\|^{(10 / 3)}} \Delta_{03}(t)+\frac{3 \varepsilon_{25} u_{1}^{T}}{2\left\|u_{1}\right\|^{4}} \Delta_{03}(t)+\frac{1}{\varepsilon_{27}}\|\alpha\|_{F}^{3} \Delta_{24}(t) .
\end{aligned}
$$

Note that

$$
\begin{aligned}
\widehat{R}_{3}^{-1} & =\frac{1}{\left\|\widehat{R}_{3}\right\|}\left(\begin{array}{cc}
R_{22} & -R_{11} \\
R_{21} & -R_{12}
\end{array}\right)=\frac{1}{\cos \phi \cos \theta}\left(\begin{array}{cc}
R_{22} & -R_{11} \\
R_{21} & -R_{12}
\end{array}\right) \\
& =\frac{1}{R_{33}}\left(\begin{array}{cc}
R_{22} & -R_{11} \\
R_{21} & -R_{12}
\end{array}\right),
\end{aligned}
$$

In fact, $\mathrm{d} R=R \times w \mathrm{~d} t$, which is

$$
\left(\begin{array}{lll}
\mathrm{d} R_{11} & \mathrm{~d} R_{12} & \mathrm{~d} R_{13} \\
\mathrm{~d} R_{21} & \mathrm{~d} R_{22} & \mathrm{~d} R_{23} \\
\mathrm{~d} R_{31} & \mathrm{~d} R_{32} & \mathrm{~d} R_{33}
\end{array}\right)=\left(\begin{array}{lll}
R_{11} & R_{12} & R_{13} \\
R_{21} & R_{22} & R_{23} \\
R_{31} & R_{32} & R_{33}
\end{array}\right)\left(\begin{array}{ccc}
0 & -r & q \\
r & 0 & -p \\
-q & p & 0
\end{array}\right) .
$$

Thus, $\mathrm{d} \widehat{R}_{3}^{-1}$ can be obtained for the controller $u_{2}$. Moreover, we can easily get that

then we have

$$
\mathrm{d} \widehat{R}_{3}^{-1}=\frac{-\mathrm{d} R_{33}}{R_{33}^{2}}\left(\begin{array}{cc}
R_{22} & -R_{11} \\
R_{21} & -R_{12}
\end{array}\right)+\frac{1}{R_{33}}\left(\begin{array}{cc}
\mathrm{d} R_{22} & -\mathrm{d} R_{11} \\
\mathrm{~d} R_{21} & -\mathrm{d} R_{12}
\end{array}\right) .
$$

$$
\mathrm{d}\left(\left(k_{5}+H_{25}+\frac{H_{24}}{4\left\|u_{1}\right\|^{(4 / 3)}}+\frac{3 \varepsilon_{25}}{4\left\|u_{1}\right\|^{2}}+\frac{1}{4 \varepsilon_{27}}\|\alpha\|_{F}^{4}\right) \widehat{R}_{3}^{-1} \bar{R}_{3 e}\right)=\Delta_{41}(t) \mathrm{d} t+\Delta_{42}(t) \widetilde{\delta}_{1} \mathrm{~d} t+\Delta_{43}(t) \mathrm{d} \beta_{1},
$$

where

$$
\begin{aligned}
\Delta_{41}(t)= & \widehat{R}_{3}^{-1} \bar{R}_{3 e} \Delta_{31}(t)+\left(k_{5}+H_{25}+\frac{H_{24}}{4\left\|u_{1}\right\|^{(4 / 3)}}+\frac{3 \varepsilon_{25}}{4\left\|u_{1}\right\|^{2}}+\frac{1}{4 \varepsilon_{27}}\|\alpha\|_{F}^{4}\right) d \widehat{R}_{3}^{-1} \bar{R}_{3 e} \\
& +\left(k_{5}+H_{25}+\frac{H_{24}}{4\left\|u_{1}\right\|^{(4 / 3)}}+\frac{3 \varepsilon_{25}}{4\left\|u_{1}\right\|^{2}}+\frac{1}{4 \varepsilon_{27}}\|\alpha\|_{F}^{4}\right) \widehat{R}_{3}^{-1} \bar{R}_{3 e 1}(t), \\
\Delta_{42}(t)= & \widehat{R}_{3}^{-1} \bar{R}_{3 e} \Delta_{32}(t)+\left(k_{5}+H_{25}+\frac{H_{24}}{4\left\|u_{1}\right\|^{(4 / 3)}}+\frac{3 \varepsilon_{25}}{4\left\|u_{1}\right\|^{2}}+\frac{1}{4 \varepsilon_{27}}\|\alpha\|_{F}^{4}\right) \widehat{R}_{3}^{-1} \bar{R}_{3 e 2}(t), \\
\Delta_{43}(t)= & \widehat{R}_{3}^{-1} \bar{R}_{3 e} \Delta_{33}(t)+\left(k_{5}+H_{25}+\frac{H_{24}}{4\left\|u_{1}\right\|^{(4 / 3)}}+\frac{3 \varepsilon_{25}}{4\left\|u_{1}\right\|^{2}}+\frac{1}{4 \varepsilon_{27}}\|\alpha\|_{F}^{4}\right) \widehat{R}_{3}^{-1} \bar{R}_{3 e 3}(t) .
\end{aligned}
$$




\section{B. The Calculation of $\mathrm{d}\left(\left(\varepsilon_{12}\left\|u_{1}\right\|^{3}\|\boldsymbol{\alpha}\|^{2}\|F(t)\|^{2}\right.\right.$}

13) $\left.\widehat{R}_{3}^{-1} \boldsymbol{\alpha}^{T} F(t)\right)$

Consider

$$
\begin{aligned}
\mathrm{d}( & \left.\frac{\varepsilon_{12}\left\|u_{1}\right\|^{3}\|\alpha\|^{2}\|F(t)\|^{2}}{3} \widehat{R}_{3}^{-1} \alpha^{T} F(t)\right) \\
= & \varepsilon_{12}\left\|u_{1}\right\|^{2} \mathrm{~d}\left\|u_{1}\right\|\|\alpha\|^{2}\|F(t)\|^{2} \widehat{R}_{3}^{-1} \alpha^{T} F(t)+\frac{2 \varepsilon_{12}}{3}\left\|u_{1}\right\|^{3}\|\alpha\| \mathrm{d}\|\alpha\|\|F(t)\|^{2} \widehat{R}_{3}^{-1} \alpha^{T} F(t) \\
& +\frac{2 \varepsilon_{12}}{3}\left\|u_{1}\right\|\|\alpha\|^{2}\|F(t)\| \mathrm{d}\|F(t)\| \widehat{R}_{3}^{-1} \alpha^{T} F(t)+\frac{\varepsilon_{12}}{3}\left\|u_{1}\right\|^{3}\|\alpha\|^{2}\|F(t)\|^{2} \mathrm{~d} \widehat{R}_{3}^{-1} \alpha^{T} F(t) \\
& +\frac{\varepsilon_{12}}{3}\left\|u_{1}\right\|^{3}\|\alpha\|^{2}\|F(t)\|^{2} \widehat{R}_{3}^{-1} \mathrm{~d} \alpha^{T} F(t)+\frac{\varepsilon_{12}}{3}\left\|u_{1}\right\|^{3}\|\alpha\|^{2}\|F(t)\|^{2} \widehat{R}_{3}^{-1} \alpha^{T} \mathrm{~d} F(t) .
\end{aligned}
$$

Hence,

$$
\begin{aligned}
& \varepsilon_{12}\left\|u_{1}\right\|^{2} \mathrm{~d}\left\|u_{1}\right\|\|\alpha\|^{2}\|F(t)\|^{2} \widehat{R}_{3}^{-1} \alpha^{T} F(t) \\
& =\frac{2 \varepsilon_{12}}{3}\left\|u_{1}\right\|^{3}\|\alpha\|\|F(t)\|^{2} \widehat{R}_{3}^{-1} \alpha^{T} F(t) \|\left(\Delta_{22}(t) \mathrm{d} t+\Delta_{23}(t) \widetilde{\delta}_{1} \mathrm{~d} t+\Delta_{24}(t) \mathrm{d} \beta_{1}\right) . \\
& \square \quad \mathrm{d} F(t)=\Delta_{401}(t) \mathrm{d} t+\Delta_{402}(t) \widetilde{\delta}_{1} \mathrm{~d} t+\Delta_{403}(t) \mathrm{d} \beta_{1},
\end{aligned}
$$

For $\left(2 \varepsilon_{12} / 3\right)\left\|u_{1}\right\|^{3}\|\alpha\|^{2}\|F(t)\| \mathrm{d}\|F(t)\| \widehat{R}_{3}^{-1} \alpha^{T} F(t)$, we first calculate the $\mathrm{d} F(t)$ :

where

$$
\begin{aligned}
\Delta_{401}(t)= & \left(\left(k_{1}+k_{2}+1\right) I+k_{1} \operatorname{Tanh}^{2}\left(e_{p}+e_{v}\right)+k_{2}\left(\operatorname{Tanh}^{2}\left(e_{v}\right)\right)\right)\left(-k_{1} \tanh \left(e_{p}+e_{v}\right)-k_{2} \tanh \left(e_{v}\right)+\frac{T_{m}}{m} \alpha \bar{R}_{3 e}\right) \\
& +k_{1}\left(I+\operatorname{Tanh}^{2}\left(e_{p}+e_{v}\right)\right) e_{v}, \\
\Delta_{402}(t)= & \left(\left(k_{1}+k_{2}+1\right) I+k_{1} \operatorname{Tanh}^{2}\left(e_{p}+e_{v}\right)+k_{2}\left(\operatorname{Tanh}^{2}\left(e_{v}\right)\right)\right), \\
\Delta_{403}(t)= & \left(\left(k_{1}+k_{2}+1\right) I+k_{1} \operatorname{Tanh}^{2}\left(e_{p}+e_{v}\right)+k_{2}\left(\operatorname{Tanh}^{2}\left(e_{v}\right)\right)\right) G_{1} .
\end{aligned}
$$

Thus, we obtain

$$
\mathrm{d}\|F(t)\|=-\frac{\mathrm{d} F(t)^{T} \cdot F(t)+F(t)^{T} \mathrm{~d} F(t)}{2 \sqrt{F(t)^{T} F(t)}}=-\frac{F(t)^{T}}{\|F(t)\|}\left(\Delta_{401}(t) \mathrm{d} t+\Delta_{402}(t) \widetilde{\delta}_{1} \mathrm{~d} t+\Delta_{403}(t) \mathrm{d} \beta_{1}\right) .
$$

Then, we drive 


$$
\begin{aligned}
& \frac{2 \varepsilon_{12}}{3}\left\|u_{1}\right\|^{3}\|\alpha\|^{2}\|F(t)\| \mathrm{d}\|F(t)\| \widehat{R}_{3}^{-1} \alpha^{T} F(t) \\
= & -\frac{2 \varepsilon_{12}}{3}\left\|u_{1}\right\|^{3}\|\alpha\|^{2}\|F(t)\| \widehat{R}_{3}^{-1} \alpha^{T} F(t)\left(\frac{F(t)^{T}}{\|F(t)\|}\left(\Delta_{401}(t) \mathrm{d} t+\Delta_{402}(t) \widetilde{\delta}_{1} \mathrm{~d} t+\Delta_{403}(t) \mathrm{d} \beta_{1}\right)\right) .
\end{aligned}
$$

In order to calculate $\left(\varepsilon_{12} / 3\right)\left\|u_{1}\right\|^{3}\|\alpha\|^{2}\|F(t)\|^{2}$

$\widehat{R}_{3}^{-1} \mathrm{~d} \alpha^{T} F(t)$, we first give the derivative of $\mathrm{d} \alpha$ as follows:

$$
\begin{aligned}
& \mathrm{d} \alpha=\left(\begin{array}{cc}
0 & 0 \\
0 & 0 \\
\frac{-\left(R_{33}+R_{33 c}\right) \mathrm{d} R_{13}+\left(R_{13}+R_{13 c}\right) \mathrm{d} R_{33}}{\left(R_{33}+R_{33 c}\right)^{2}} & \frac{-\left(R_{33}+R_{33 c}\right) \mathrm{d} R_{23}+\left(R_{23}+R_{23 c}\right) \mathrm{d} R_{33}}{\left(R_{33}+R_{33 c}\right)^{2}}
\end{array}\right) \\
& +\left(\begin{array}{cc}
0 & 0 \\
0 & 0 \\
\frac{-\left(R_{33}+R_{33 c}\right) \mathrm{d} R_{13 c}+\left(R_{13}+R_{13 c}\right) \mathrm{d} R_{33 c}}{\left(R_{33}+R_{33 c}\right)^{2}} & \frac{-\left(R_{33}+R_{33 c}\right) \mathrm{d} R_{23 c}+\left(R_{23}+R_{23 c}\right) \mathrm{d} R_{33 c}}{\left(R_{33}+R_{33 c}\right)^{2}}
\end{array}\right) .
\end{aligned}
$$

Due to

$$
\begin{aligned}
& \frac{-\left(R_{33}+R_{33 c}\right) \mathrm{d} R_{13 c}+\left(R_{13}+R_{13 c}\right) \mathrm{d} R_{33 c}}{\left(R_{33}+R_{33 c}\right)^{2}}=\Delta_{501}(t)+\Delta_{502}(t) \widetilde{\delta}_{1}+\Delta_{503}(t) \mathrm{d} \beta_{1}, \\
& \frac{-\left(R_{33}+R_{33 c}\right) \mathrm{d} R_{23 c}+\left(R_{23}+R_{23 c}\right) \mathrm{d} R_{33 c}}{\left(R_{33}+R_{33 c}\right)^{2}}=\Delta_{511}(t)+\Delta_{512}(t) \widetilde{\delta}_{1}+\Delta_{513}(t) \mathrm{d} \beta_{1},
\end{aligned}
$$

where

$$
\begin{aligned}
& \Delta_{501}(t)=\frac{1}{\left(R_{33}+R_{33 c}\right)^{2}}\left(R_{33}+R_{33 c} 0-\left(R_{13}+R_{13 c}\right)\right) \Delta_{01}(t) \text {, } \\
& \Delta_{502}(t)=\frac{1}{\left(R_{33}+R_{33 c}\right)^{2}}\left(R_{33}+R_{33 c} 0-\left(R_{13}+R_{13 c}\right)\right) \Delta_{02}(t) \text {, } \\
& \Delta_{503}(t)=\frac{1}{\left(R_{33}+R_{33 c}\right)^{2}}\left(R_{33}+R_{33 c} 0-\left(R_{13}+R_{13 c}\right)\right) \Delta_{03}(t) \text {, } \\
& \Delta_{511}(t)=\frac{1}{\left(R_{33}+R_{33 c}\right)^{2}}\left(R_{33}+R_{33 c} 0-\left(R_{23}+R_{23 c}\right)\right) \Delta_{01}(t) \text {, } \\
& \Delta_{512}(t)=\frac{1}{\left(R_{33}+R_{33 c}\right)^{2}}\left(R_{33}+R_{33 c} 0-\left(R_{23}+R_{23 c}\right)\right) \Delta_{02}(t) \text {, } \\
& \Delta_{513}(t)=\frac{1}{\left(R_{33}+R_{33 c}\right)^{2}}\left(R_{33}+R_{33 c} 0-\left(R_{23}+R_{23 c}\right)\right) \Delta_{03}(t) \text {. }
\end{aligned}
$$


Then, $\mathrm{d} \alpha$ is represented as

where

$$
\mathrm{d} \alpha=\Delta_{51}(t)+\Delta_{52}\left(t, \widetilde{\delta}_{1}\right)+\Delta_{53}(t) \mathrm{d} \beta_{1},
$$

$$
\begin{aligned}
& \alpha_{2}=\left(\begin{array}{cc}
0 & 0 \\
0 & 0 \\
\frac{-\left(R_{33}+R_{33 c}\right) \mathrm{d} R_{13}+\left(R_{13}+R_{13 c}\right) \mathrm{d} R_{33}}{\left(R_{33}+R_{33 c}\right)^{2}} \frac{-\left(R_{33}+R_{33 c}\right) \mathrm{d} R_{23}+\left(R_{23}+R_{23 c}\right) \mathrm{d} R_{33}}{\left(R_{33}+R_{33 c}\right)^{2}}
\end{array}\right), \\
& \alpha_{31}=\left(\begin{array}{ll}
0 & 0 \\
0 & 0 \\
1 & 0
\end{array}\right), \\
& \alpha_{32}=\left(\begin{array}{ll}
0 & 0 \\
0 & 0 \\
0 & 1
\end{array}\right), \\
& \Delta_{51}=\alpha_{2}+\alpha_{31} \Delta_{501}(t)+\alpha_{32} \Delta_{511}(t), \\
& \Delta_{52}\left(t, \widetilde{\delta}_{1}\right)=\Delta_{502}(t) \widetilde{\delta}_{1} \alpha_{31}+\Delta_{512}(t) \widetilde{\delta}_{1} \alpha_{32}, \\
& \Delta_{53}=\alpha_{31} \Delta_{503}(t)+\alpha_{32} \Delta_{513}(t) .
\end{aligned}
$$

Hence, we can obtain that

$$
\frac{\varepsilon_{12}}{3}\left\|u_{1}\right\|^{3}\|\alpha\|^{2}\|F(t)\|^{2} \widehat{R}_{3}^{-1} \mathrm{~d} \alpha^{T} F(t)=\frac{\varepsilon_{12}}{3}\left\|u_{1}\right\|^{3}\|\alpha\|^{2}\|F(t)\|^{2} \widehat{R}_{3}^{-1}\left(\Delta_{51}(t)+\Delta_{52}\left(t, \widetilde{\delta}_{1}\right)+\Delta_{53}(t) \mathrm{d} \beta_{1}\right)^{T} F(t) .
$$

For the part of $\left(\varepsilon_{12} / 3\right)\left\|u_{1}\right\|^{3}\|\alpha\|^{2}\|F(t)\|^{2} \widehat{R}_{3}^{-1} \alpha^{T} \mathrm{~d} F(t)$, we have

$$
\frac{\varepsilon_{12}}{3}\left\|u_{1}\right\|^{3}\|\alpha\|^{2}\|F(t)\|^{2} \widehat{R}_{3}^{-1} \alpha^{T} \mathrm{~d} F(t)=\frac{\varepsilon_{12}}{3}\left\|u_{1}\right\|^{3}\|\alpha\|^{2}\|F(t)\|^{2} \widehat{R}_{3}^{-1} \alpha^{T}\left(\Delta_{401}(t) \mathrm{d} t+\Delta_{402}(t) \widetilde{\delta}_{1} \mathrm{~d} t+\Delta_{403}(t) \mathrm{d} \beta_{1}\right)
$$

Hence,

$$
d\left(\frac{\varepsilon_{12}\left\|u_{1}\right\|^{3}\|\alpha\|^{2}\|F(t)\|^{2}}{3} \widehat{R}_{3}^{-1} \alpha^{T} F(t)\right)=\Delta_{61}(t) \mathrm{d} t+\Delta_{621}(t) \widetilde{\delta}_{1} \mathrm{~d} t+\Delta_{622}\left(t, \widetilde{\delta}_{1}\right) \mathrm{d} t+\Delta_{63}(t) \mathrm{d} \beta_{1}
$$

where 
$\Delta_{61}(t)=-\varepsilon_{12}\left\|u_{1}\right\|\|\alpha\|^{2}\|F(t)\|^{2} \widehat{R}_{3}^{-1} \alpha^{T} F(t) u_{1}^{T} \Delta_{01}(t)+\frac{2 \varepsilon_{12}}{3}\left\|u_{1}\right\|^{3}\|\alpha\|\|F(t)\|^{2} \widehat{R}_{3}^{-1} \alpha^{T} F(t) \Delta_{22}(t)$

$-\frac{2 \varepsilon_{12}}{3}\left\|u_{1}\right\|^{3}\|\alpha\|^{2} \widehat{R}_{3}^{-1} \alpha^{T} F(t) F(t)^{T} \Delta_{401}(t)+\frac{\varepsilon_{12}}{3}\left\|u_{1}\right\|^{3}\|\alpha\|^{2}\|F(t)\|^{2} \mathrm{~d} \widehat{R}_{3}^{-1} \alpha^{T} F(t)$

$+\frac{\varepsilon_{12}}{3}\left\|u_{1}\right\|^{3}\|\alpha\|^{2}\|F(t)\|^{2} \widehat{R}_{3}^{-1} \Delta_{51}(t)^{T} F(t)+\frac{\varepsilon_{12}}{3}\left\|u_{1}\right\|^{3}\|\alpha\|^{2}\|F(t)\|^{2} \widehat{R}_{3}^{-1} \alpha^{T} \Delta_{401}(t)$,

$\Delta_{621}(t)=-\varepsilon_{12}\left\|u_{1}\right\|\|\alpha\|^{2}\|F(t)\|^{2} \widehat{R}_{3}^{-1} \alpha^{T} F(t) u_{1}^{T} \Delta_{02}(t)+\frac{2 \varepsilon_{12}}{3}\left\|u_{1}\right\|^{3}\|\alpha\|\|F(t)\|^{2} \widehat{R}_{3}^{-1} \alpha^{T} F(t) \Delta_{23}(t)$

$-\frac{2 \varepsilon_{12}}{3}\left\|u_{1}\right\|^{3}\|\alpha\|^{2} \widehat{R}_{3}^{-1} \alpha^{T} F(t) F(t)^{T} \Delta_{402}(t)$

$\Delta_{63}(t)=-\varepsilon_{12}\left\|u_{1}\right\|\|\alpha\|^{2}\|F(t)\|^{2} \widehat{R}_{3}^{-1} \alpha^{T} F(t) u_{1}^{T} \Delta_{03}(t)+\frac{2 \varepsilon_{12}}{3}\left\|u_{1}\right\|^{3}\|\alpha\|\|F(t)\|^{2} \widehat{R}_{3}^{-1} \alpha^{T} F(t) \Delta_{24}(t)$

$-\frac{2 \varepsilon_{12}}{3}\left\|u_{1}\right\|^{3}\|\alpha\|^{2} \widehat{R}_{3}^{-1} \alpha^{T} F(t) F(t)^{T} \Delta_{403}(t)+\frac{\varepsilon_{12}}{3}\left\|u_{1}\right\|^{3}\|\alpha\|^{2}\|F(t)\|^{2} \widehat{R}_{3}^{-1} \Delta_{53}(t)^{T} F(t)$

$+\frac{\varepsilon_{12}}{3}\left\|u_{1}\right\|^{3}\|\alpha\|^{2}\|F(t)\|^{2} \widehat{R}_{3}^{-1} \alpha^{T} \Delta_{403}(t)$,

$\Delta_{622}\left(t, \widetilde{\delta}_{1}\right)=\frac{\varepsilon_{12}}{3}\left\|u_{1}\right\|^{3}\|\alpha\|^{2}\|F(t)\|^{2} \widehat{R}_{3}^{-1} \Delta_{52}\left(t, \widetilde{\delta}_{1}\right)^{T} F(t)$.

C. The Calculation of $\mathrm{d}\left(\left(\varepsilon_{232} / 2\left\|u_{1}\right\|^{4}\right)\left(e_{v}^{T} e_{v}\right)^{2}\right.$

$\left.\widehat{R}_{3}^{-1} \bar{R}_{3 e}\right)$

Consider

$$
\begin{aligned}
\mathrm{d}\left(\frac{\varepsilon_{232}}{2\left\|u_{1}\right\|^{4}}\left(e_{v}^{T} e_{v}\right)^{2} \widehat{R}_{3}^{-1} \bar{R}_{3 e}\right)= & \mathrm{d} \frac{\varepsilon_{232}}{2\left\|u_{1}\right\|^{4}}\left(e_{v}^{T} e_{v}\right)^{2} \widehat{R}_{3}^{-1} \bar{R}_{3 e}+\frac{\varepsilon_{232}}{2\left\|u_{1}\right\|^{4}} \mathrm{~d}\left(e_{v}^{T} e_{v}\right)^{2} \widehat{R}_{3}^{-1} \bar{R}_{3 e} \\
& +\frac{\varepsilon_{232}}{2\left\|u_{1}\right\|^{4}}\left(e_{v}^{T} e_{v}\right)^{2} \mathrm{~d} \widehat{R}_{3}^{-1} \bar{R}_{3 e}+\frac{\varepsilon_{232}}{2\left\|u_{1}\right\|^{4}}\left(e_{v}^{T} e_{v}\right)^{2} \widehat{R}_{3}^{-1} \mathrm{~d} \bar{R}_{3 e} .
\end{aligned}
$$

For the derivatives of those teams, we have

$$
\begin{aligned}
\mathrm{d} \frac{\varepsilon_{232}}{2\left\|u_{1}\right\|^{4}}\left(e_{v}^{T} e_{v}\right)^{2} \widehat{R}_{3}^{-1} \bar{R}_{3 e}= & \frac{4 \varepsilon_{232}}{2\left\|u_{1}\right\|^{6}}\left(e_{v}^{T} e_{v}\right)^{2} \widehat{R}_{3}^{-1} \bar{R}_{3 e} u_{1}^{T}\left(\Delta_{01}(t) \mathrm{d} t+\Delta_{02}(t) \widetilde{\delta}_{1} \mathrm{~d} t+\Delta_{03}(t) \mathrm{d} \beta_{1}\right), \\
\frac{\varepsilon_{232}}{2\left\|u_{1}\right\|^{4}} \mathrm{~d}\left(e_{v}^{T} e_{v}\right)^{2} \widehat{R}_{3}^{-1} \bar{R}_{3 e}= & \frac{4 \varepsilon_{232}}{2\left\|u_{1}\right\|^{4}} \widehat{R}_{3}^{-1} \bar{R}_{3 e} e_{v}^{T} e_{v} e_{v}^{T}\left(-k_{1} \tanh \left(e_{p}+e_{v}\right)-k_{2} \tanh \left(e_{v}\right)\right. \\
& \left.+\frac{T_{m}}{m} \alpha \bar{R}_{3 e}+\widetilde{\delta}_{1}+G_{1} \mathrm{~d} \beta_{1}\right), \\
\frac{\varepsilon_{232}}{2\left\|u_{1}\right\|^{4}}\left(e_{v}^{T} e_{v}\right)^{2} \widehat{R}_{3}^{-1} \mathrm{~d} \bar{R}_{3 e}= & \frac{\varepsilon_{232}}{2\left\|u_{1}\right\|^{4}}\left(e_{v}^{T} e_{v}\right)^{2} \widehat{R}_{3}^{-1}\left(\bar{R}_{3 e 1}(t) \mathrm{d} t+\bar{R}_{3 e 2}(t) \widetilde{\delta}_{1} \mathrm{~d} t+\bar{R}_{3 e 3}(t) \mathrm{d} \beta_{1}\right) .
\end{aligned}
$$

Therefore, one has 


$$
\mathrm{d}\left(\frac{\varepsilon_{232}}{2\left\|u_{1}\right\|^{4}}\left(e_{v}^{T} e_{v}\right)^{2} \widehat{R}_{3}^{-1} \bar{R}_{3 e}\right)=\Delta_{71}(t) \mathrm{d} t+\Delta_{72}(t) \widetilde{\delta}_{1} \mathrm{~d} t+\Delta_{73}(t) \mathrm{d} \beta_{1}
$$

where

$$
\begin{aligned}
\Delta_{71}(t)= & \frac{4 \varepsilon_{232}}{2\left\|u_{1}\right\|^{6}}\left(e_{v}^{T} e_{v}\right)^{2} \widehat{R}_{3}^{-1} \bar{R}_{3 e} u_{1}^{T} \Delta_{01}(t)+\frac{4 \varepsilon_{232}}{2\left\|u_{1}\right\|^{4}} \widehat{R}_{3}^{-1} \bar{R}_{3 e} e_{v}^{T} e_{v} e_{v}^{T}\left(-k_{1} \tanh \left(e_{p}+e_{v}\right)-k_{2} \tanh \left(e_{v}\right)\right) \\
& +\frac{T_{m}}{m} \alpha \bar{R}_{3 e}+\frac{\varepsilon_{232}}{2\left\|u_{1}\right\|^{4}}\left(e_{v}^{T} e_{v}\right)^{2} \mathrm{~d} \widehat{R}_{3}^{-1} \bar{R}_{3 e}+\frac{\varepsilon_{232}}{2\left\|u_{1}\right\|^{4}}\left(e_{v}^{T} e_{v}\right)^{2} \widehat{R}_{3}^{-1} \bar{R}_{3 e 1}(t), \\
\Delta_{72}(t)= & \frac{4 \varepsilon_{232}}{2\left\|u_{1}\right\|^{6}}\left(e_{v}^{T} e_{v}\right)^{2} \widehat{R}_{3}^{-1} \bar{R}_{3 e} u_{1}^{T} \Delta_{02}(t)+\frac{4 \varepsilon_{232}}{2\left\|u_{1}\right\|^{4}} \widehat{R}_{3}^{-1} \bar{R}_{3 e} e_{v}^{T} e_{v} e_{v}^{T}+\frac{\varepsilon_{232}}{2\left\|u_{1}\right\|^{4}}\left(e_{v}^{T} e_{v}\right)^{2} \widehat{R}_{3}^{-1} \bar{R}_{3 e 2}(t), \\
\Delta_{73}(t)= & \frac{4 \varepsilon_{232}}{2\left\|u_{1}\right\|^{6}}\left(e_{v}^{T} e_{v}\right)^{2} \widehat{R}_{3}^{-1} \bar{R}_{3 e} u_{1}^{T} \Delta_{03}(t)+\frac{4 \varepsilon_{232}}{2\left\|u_{1}\right\|^{4}} \widehat{R}_{3}^{-1} \bar{R}_{3 e} e_{v}^{T} e_{v} e_{v}^{T} G_{1}+\frac{\varepsilon_{232}}{2\left\|u_{1}\right\|^{4}}\left(e_{v}^{T} e_{v}\right)^{2} \widehat{R}_{3}^{-1} \bar{R}_{3 e 3}(t) .
\end{aligned}
$$

\section{The Calculation of $\mathrm{d}\left(\left(\varepsilon_{11}\|F(t)\|^{2} / 3\left\|u_{1}\right\|\right) \widehat{R}_{3}^{-1}\right.$}

\section{$\left.E_{0} F(t)\right)$}

Consider

$$
\begin{aligned}
\mathrm{d}\left(\frac{\varepsilon_{11}\|F(t)\|^{2}}{3\left\|u_{1}\right\|} \widehat{R}_{3}^{-1} E_{0} F(t)\right)= & \mathrm{d} \frac{\varepsilon_{11}}{3\left\|u_{1}\right\|}\|F(t)\|^{2} \widehat{R}_{3}^{-1} E_{0} F(t)+\frac{\varepsilon_{11}}{3\left\|u_{1}\right\|} \mathrm{d}\|F(t)\|^{2} \widehat{R}_{3}^{-1} E_{0} F(t) \\
& +\frac{\varepsilon_{11}}{3\left\|u_{1}\right\|}\|F(t)\|^{2} \mathrm{~d} \widehat{R}_{3}^{-1} E_{0} F(t)+\frac{\varepsilon_{11}}{3\left\|u_{1}\right\|}\|F(t)\|^{2} \widehat{R}_{3}^{-1} E_{0} \mathrm{~d} F(t) .
\end{aligned}
$$

For the derivatives of those teams, we have

$$
\begin{aligned}
\mathrm{d} \frac{\varepsilon_{11}}{3\left\|u_{1}\right\|}\|F(t)\|^{2} \widehat{R}_{3}^{-1} E_{0} F(t) & =\frac{\varepsilon_{11}}{3\left\|u_{1}\right\|^{3}}\|F(t)\|^{2} \widehat{R}_{3}^{-1} E_{0} F(t) u_{1}^{T}\left(\Delta_{01}(t) \mathrm{d} t+\Delta_{02}(t) \widetilde{\delta}_{1} \mathrm{~d} t+\Delta_{03}(t) \mathrm{d} \beta_{1}\right) \\
\frac{\varepsilon_{11}}{3\left\|u_{1}\right\|} \mathrm{d}\|F(t)\|^{2} \widehat{R}_{3}^{-1} E_{0} F(t) & =\frac{2 \varepsilon_{11}}{3\left\|u_{1}\right\|}\|F(t)\| \widehat{R}_{3}^{-1} E_{0} F(t) \mathrm{d}\|F(t)\|, \\
& =-\frac{2 \varepsilon_{11}}{3\left\|u_{1}\right\|}\|F(t)\| \widehat{R}_{3}^{-1} E_{0} F(t) \frac{F(t)^{T}}{\|F(t)\|}\left(\Delta_{401}(t) \mathrm{d} t+\Delta_{402}(t) \widetilde{\delta}_{1} d t+\Delta_{403}(t) \mathrm{d} \beta_{1}\right), \\
\frac{\varepsilon_{11}}{3\left\|u_{1}\right\|}\|F(t)\|^{2} \widehat{R}_{3}^{-1} E_{0} \mathrm{~d} F(t) & =\frac{\varepsilon_{11}}{3\left\|u_{1}\right\|}\|F(t)\|^{2} \widehat{R}_{3}^{-1} E_{0}\left(\Delta_{401}(t) \mathrm{d} t+\Delta_{402}(t) \widetilde{\delta}_{1} \mathrm{~d} t+\Delta_{403}(t) \mathrm{d} \beta_{1}\right) .
\end{aligned}
$$

Hence, we deduce

$$
\mathrm{d}\left(\frac{\varepsilon_{11}\|F(t)\|^{2}}{3\left\|u_{1}\right\|} \widehat{R}_{3}^{-1} E_{0} F(t)\right)=\Delta_{81}(t) \mathrm{d} t+\Delta_{82}(t) \widetilde{\delta}_{1} \mathrm{~d} t+\Delta_{83}(t) \mathrm{d} \beta_{1},
$$


where

$$
\begin{aligned}
& \Delta_{81}(t)=\frac{\varepsilon_{11}}{3\left\|u_{1}\right\|^{3}}\|F(t)\|^{2} \widehat{R}_{3}^{-1} E_{0} F(t) u_{1}^{T} \Delta_{01}(t)-\frac{2 \varepsilon_{11}}{3\left\|u_{1}\right\|^{-1}} \widehat{R}_{3}^{-1} E_{0} F(t) F(t)^{T} \Delta_{401}(t) \\
& +\frac{\varepsilon_{11}}{3\left\|u_{1}\right\|}\|F(t)\|^{2} \mathrm{~d} \widehat{R}_{3}^{-1} E_{0} F(t)+\frac{\varepsilon_{11}}{3\left\|u_{1}\right\|}\|F(t)\|^{2} \widehat{R}_{3}^{-1} E_{0} \Delta_{401}(t) \\
& \Delta_{82}(t)=\frac{\varepsilon_{11}}{3\left\|u_{1}\right\|}\|F(t)\|^{2} \widehat{R}_{3}^{-1} E_{0} F(t) u_{1}^{T} \Delta_{02}(t)-\frac{2 \varepsilon_{11}}{3\left\|u_{1}\right\|} \widehat{R}_{3}^{-1} E_{0} F(t) F(t)^{T} \Delta_{402}(t)+\frac{\varepsilon_{11}}{3\left\|u_{1}\right\|^{\|}}\|F(t)\|^{2} \widehat{R}_{3}^{-1} E_{0} \Delta_{402}(t), \\
& \Delta_{83}(t)=\frac{\varepsilon_{11}}{3\left\|u_{1}\right\|}\|F(t)\|^{2} \widehat{R}_{3}^{-1} E_{0} F(t) u_{1}^{T} \Delta_{03}(t)-\frac{2 \varepsilon_{11}}{3\left\|u_{1}\right\|} \widehat{R}_{3}^{-1} E_{0} F(t) F(t)^{T} \Delta_{403}(t)+\frac{\varepsilon_{11}}{3\left\|u_{1}\right\|^{3}}\|F(t)\|^{2} \widehat{R}_{3}^{-1} E_{0} \Delta_{403}(t) .
\end{aligned}
$$

\section{E. The Calculation of $\mathrm{d} w_{1 c}$}

$\mathrm{d} w_{1 c}$ can be obtained as

$$
\mathrm{d} w_{1 c}=W_{1 c 1}(t) \mathrm{d} t+W_{1 c 2}(t) \widetilde{\delta}_{1} \mathrm{~d} t+\Delta_{622}\left(t, \widetilde{\delta}_{1}\right) \mathrm{d} t+W_{1 c 3}(t) \mathrm{d} \beta_{1},
$$

where

$$
\begin{aligned}
& W_{1 c 1}(t)=\Delta_{41}(t)+\Delta_{61}(t)+\Delta_{71}(t)+\Delta_{81}(t), \\
& W_{1 c 2}(t)=\Delta_{42}(t)+\Delta_{621}(t)+\Delta_{72}(t)+\Delta_{82}(t), \\
& W_{1 c 3}(t)=\Delta_{43}(t)+\Delta_{63}(t)+\Delta_{73}(t)+\Delta_{83}(t) .
\end{aligned}
$$

\section{F. The Calculation of $\mathrm{d} r_{c}$}

For $r_{c}$, we have

$$
r_{c}=-\frac{\cos \theta}{\cos \phi}\left(\frac{\sin \phi}{\cos \theta} w_{1 c}-\mathrm{d} \psi_{r}+k_{3} e_{\psi}\right)=-\tan \phi\left(E_{2} q_{c}-\mathrm{d} \psi_{r}+k_{3} e_{\psi}\right)
$$

$$
\mathrm{d} r_{c}=W_{r c 1}(t) \mathrm{d} t+W_{r c 2}(t) \widetilde{\delta}_{1} \mathrm{~d} t+W_{r c 20}\left(t, \widetilde{\delta}_{1}\right) \mathrm{d} t+W_{r c 3}(t) \mathrm{d} \beta_{1},
$$

where $E_{2}=\left(\begin{array}{ll}0 & 1\end{array}\right)$. Define $\Omega=\left(\begin{array}{lll}\phi & \theta & \psi\end{array}\right)$, then the derivative of $\Omega$ is

where

$$
\dot{\Omega}=\left(\begin{array}{ccc}
1 & S \phi T \theta & C \phi T \theta \\
0 & C \phi & -S \phi \\
0 & \frac{S \phi}{C \theta} & \frac{C \phi}{C \theta}
\end{array}\right)\left(\begin{array}{l}
p \\
q \\
r
\end{array}\right) \text {, }
$$

$$
\begin{aligned}
W_{r c 1}(t)= & -\left(E_{2} w_{1 c}-\mathrm{d} \psi_{r}+k_{3} e_{\psi}\right)\left(1+\tan ^{2} \phi\right) \mathrm{d} \phi \\
& -\tan \phi E_{2} W_{1 c 1}(t) \mathrm{d} t+\tan \phi d \dot{\psi}_{r} \\
& -\tan \phi k_{6}\left(-k_{6} e_{\psi}+E_{1} e_{w}\right), \\
W_{r c 2}(t)= & -\tan \phi E_{2} W_{1 c 2}(t), \\
W_{r c 20}\left(t, \widetilde{\delta}_{1}\right)= & -\tan \phi E_{2} \Delta_{622}\left(t, \widetilde{\delta}_{1}\right), \\
W_{r c 3}(t)= & -\tan \phi E_{2} W_{1 c 3} .
\end{aligned}
$$




\section{G. The Calculation of $\mathrm{d} w_{c}$}

The derivative of $w_{c}$ is obtained as follows:

$$
\mathrm{d} w_{c}=\left(\begin{array}{c}
\mathrm{d} w_{1 c} \\
\mathrm{~d} r_{c}
\end{array}\right)=W_{c 1}(t) \mathrm{d} t+W_{c 2}(t) \widetilde{\delta}_{1}+W_{c 20}\left(t, \widetilde{\delta}_{1}\right)+W_{c 3}(t) \mathrm{d} \beta_{1}
$$

Notice that

$$
W_{c 20}\left(t, \widetilde{\delta}_{1}\right)=\left(\begin{array}{c}
\Delta_{622}\left(t, \widetilde{\delta}_{1}\right) \\
W_{r c 20}\left(t, \widetilde{\delta}_{1}\right)
\end{array}\right)=W_{c 21}(t)\left(\Delta_{502}(t) \widetilde{\delta}_{1} \alpha_{31}+\Delta_{512}(t) \widetilde{\delta}_{1} \alpha_{32}\right)^{T} F(t)
$$

with

which implies that

$$
W_{c 21}(t)=\left(\begin{array}{c}
1 \\
-\tan \phi E_{2}
\end{array}\right) \frac{\varepsilon_{12}}{3}\left\|u_{1}\right\|^{3}\|\alpha\|^{2}\|F(t)\|^{2} \widehat{R}_{3}^{-1},
$$

$$
\left\|W_{c 20}\left(t, \widetilde{\delta}_{1}\right)\right\| \leq\left\|W_{c 21}(t)\right\|\left\|\Delta_{502}(t)\right\|\left\|\widetilde{\delta}_{1}\right\|\left\|\alpha_{31}\right\|\|F(t)\|+\left\|W_{c 21}(t)\right\|\left\|\Delta_{502}(t)\right\|\left\|\widetilde{\delta}_{1}\right\|\left\|\alpha_{31}\right\|\|F(t)\| .
$$

\section{Data Availability}

No data were used to support this study.

\section{Conflicts of Interest}

The authors declare that they have no conflicts of interest.

\section{Acknowledgments}

This work was supported by the National Natural Science Foundation of China (Grant nos. 12101481 and 62103327).

\section{References}

[1] A. Isidori, Nonlinear Control Systems II, Springer, London, UK, 1999.

[2] H. K. Khalil, Nonlinear Systems, Prentice-Hall, Hoboken, NJ, USA, Third edition, 2002.

[3] D. Comminiello and J. C. Príncipe, Adaptive Learning Methods for Nonlinear System Modeling, Butterworth-Heinemann, Oxford, UK, 2018.

[4] A. Wang, L. Liu, J. Qiu, and G. Feng, "Event-triggered robust adaptive fuzzy control for a class of nonlinear systems," IEEE Transactions on Fuzzy Systems, vol. 27, no. 8, pp. 1648-1658, 2019.

[5] W. Meng, Q. Yang, and Y. Sun, "Adaptive neural control of nonlinear MIMO systems with time-varying output constraints," IEEE Transactions on Neural Networks and Learning Systems, vol. 26, no. 5, pp. 1074-1085, 2015.
[6] Z. Zeng Lian Liu and J. Svoboda, "A new control scheme for nonlinear systems with disturbances," IEEE Transactions on Control Systems Technology, vol. 14, no. 1, pp. 176-181, 2006.

[7] T. Wang, J. Wu, Y. Wang, and M. Ma, "Adaptive fuzzy tracking control for a class of strict-feedback nonlinear systems with time-varying input delay and full state constraints," IEEE Transactions on Fuzzy Systems, vol. 28, no. 12, pp. 3432-3441, 2020.

[8] P. Zhou, L. Zhang, S. Zhang, and A. F. Alkhateeb, "Observerbased adaptive fuzzy finite-time control design with prescribed performance for switched pure-feedback nonlinear systems," IEEE Access, vol. 9, pp. 69481-69491, 2021.

[9] W. Wang, R. Postoyan, D. Nesic, and W. P. M. H. Heemels, "Periodic event-triggered control for nonlinear networked control systems," IEEE Transactions on Automatic Control, vol. 65 , no. 2, pp. $620-635,2020$.

[10] Y. Wu and X.-J. Xie, "Robust adaptive control for stateconstrained nonlinear systems with input saturation and unknown control direction," IEEE Transactions on Systems, Man, and Cybernetics: Systems, vol. 51, no. 2, pp. 1192-1202, 2021.

[11] X.-H. Chang, G.-H. Yang, and H. Wang, "Observer-based Ho-control for discrete-time T-S fuzzy systems," International Journal of Systems Science, vol. 42, no. 10, pp. 1801-1809, 2011.

[12] Y. Chang, P. Zhou, B. Niu et al., "Switched-observer-based adaptive output-feedback control design with unknown gain for pure-feedback switched nonlinear systems via average dwell time," International Journal of Systems Science, vol. 52, no. 9, pp. 1731-1745, 2021.

[13] Z. Chen, B. Niu, X. Zhao, L. Zhang, and N. Xu, "Model-Based adaptive event-Triggered control of nonlinear continuous- 
time systems," Applied Mathematics and Computation, vol. 408, p. 126330, 2021.

[14] Y. Yang, Helicopter Flight Control, National Defense Industry Press, Beijing, China, 3rd edition, 2015.

[15] G. Padfield, Helicopter Flight Dynamics: The Theory and Application of Flying Qualities and Simulation Modelling, Blackwell, Oxford, UK, 2007.

[16] I. A. Raptis, K. P. Valavanis, and G. J. Vachtsevanos, "Linear tracking control for small-scale unmanned helicopters," IEEE Transactions on Control Systems Technology, vol. 20, no. 4, pp. 995-1010, 2012.

[17] Z. Fang, H. Tian, and P. Li, "Probabilistic robust linear parameter-varying control of a small helicopter using iterative scenario approach," IEEE/CAA Journal of Automatica Sinica, vol. 2, no. 1, pp. 85-93, 2015.

[18] M. Chen and Y. Li, "Model reference resilient control for the helicopter with time-varying disturbance," International Journal of Robust and Nonlinear Control, vol. 29, no. 15, pp. 5095-5117, 2019.

[19] Y. Li, M. Chen, T. Li, and H. Wang, "Tracking control for the helicopter with time-varying disturbance and input stochastic perturbation," Proceedings of the Institution of Mechanical Engineers - Part G: Journal of Aerospace Engineering, vol. 234, no. 4, pp. 961-976, 2020.

[20] Y. Zou, "Adaptive trajectory tracking control approach for a model-scaled helicopter," Nonlinear Dynamics, vol. 83, no. 4, pp. 2171-2181, 2016.

[21] P. Marantos, C. P. Bechlioulis, and K. J. Kyriakopoulos, "Robust trajectory tracking control for small-scale unmanned helicopters with model uncertainties," IEEE Transactions on Control Systems Technology, vol. 25, no. 6, pp. 2010-2021, 2017.

[22] X. Wang, X. Yu, S. Li, and J. Liu, "Composite block backstepping trajectory tracking control for disturbed unmanned helicopters," Aerospace Science and Technology, vol. 85, pp. 386-398, 2019.

[23] Y. Huang, M. Zhu, Z. Zheng, and M. Feroskhan, "Fixed-time autonomous shipboard landing control of a helicopter with external disturbances," Aerospace Science and Technology, vol. 84, pp. 18-30, 2019.

[24] T. Jiang, D. Lin, and T. Song, "Finite-time control for smallscale unmanned helicopter with disturbances," Nonlinear Dynamics, vol. 96, no. 3, pp. 1747-1763, 2019.

[25] I. Ullah and H. Pei, "Sliding mode tracking control for unmanned helicopter using extended disturbance observer," Archives of Control Sciences, vol. 29, no. 1, pp. 169-199, 2019.

[26] R. Ma, L. Ding, and H. Wu, "Dynamic decoupling control optimization for a small-scale unmanned helicopter," Journal of Robotics, vol. 2018, Article ID 9897684, 12 pages, 2018.

[27] Y. Li, M. Chen, S. S. Ge, and D. Li, "Anti-disturbance control for attitude and altitude systems of the helicopter under random disturbances," Aerospace Science and Technology, vol. 96, p. 105561, 2020.

[28] X. Mao and C. Yuan, Stochastic Differential Equations with Markovian Switching, Imperial College Press, London, UK, 2006. 